\title{
How message similarity shapes the timecourse of sentence formulation
}

\author{
Agnieszka E. Konopka ${ }^{\mathrm{a}, \mathrm{b}, *}$, Stefanie E. Kuchinsky ${ }^{\mathrm{c}}$ \\ ${ }^{a}$ Max Planck Institute for Psycholinguistics, The Netherlands \\ ${ }^{\mathrm{b}}$ Donders Center for Brain, Cognition and Behavior, Radboud Universiteit Nijmegen, The Netherlands \\ ${ }^{\mathrm{c}}$ Center for Advanced Study of Language, University of Maryland, College Park, MD, United States
}

\section{A R T I C L E I N F O}

\section{Article history:}

Received 23 December 2013

revision received 1 April 2015

\section{Keywords:}

Incrementality

Sentence formulation

Semantic boost in structural priming

\begin{abstract}
A B S T R A C T
Transforming a preverbal message into an utterance (e.g., The swimmer is pushing the papar$a z z o$ ) requires conceptual and linguistic encoding. Two experiments tested whether the timecourse of sentence formulation is shaped jointly or independently by message-level and sentence-level processes. Eye-tracked speakers described pictures of simple events with verb-medial (SVO/OVS) and verb-initial (VSO/aux-OVS) sentences in Dutch. To assess effects of message-level and sentence-level variables on formulation, the experiments manipulated the ease of relational encoding at both levels: target events were preceded by conceptually similar or dissimilar prime events (event primes) that increased speakers' familiarity with the action shown in the target event (e.g., pushing), and the prime events were accompanied by recorded active or passive descriptions (structural primes) that facilitated generation of suitable linguistic structures on target trials. The results showed effects of both types of primes on the form of target descriptions and on formulation. Speakers repeated the primed structures more often when target events were conceptually similar to the prime events. Importantly, conceptual similarity constrained the effects of structural primes on the timecourse of formulation: speakers showed more consistent deployment of attention to the two characters during linguistic encoding in structurally primed than unprimed active sentences, but conceptual familiarity reduced the priming effects in eye movements. Thus familiarity with message-level information can change how speakers express their messages and, during formulation, can provide conceptual guidance that supersedes effects of sentence-level variables. Effects of the event primes were stronger in VSO sentences, where early verb placement explicitly required early encoding of relational information, suggesting that linear word order can also constrain message-level influences on formulation.
\end{abstract}

(c) 2015 Elsevier Inc. All rights reserved.

\section{Introduction}

Speaking involves a complex series of conceptual and linguistic encoding processes (Levelt, 1989). Many of the debates in psycholinguistics have centered on questions

\footnotetext{
* Corresponding author at: Max Planck Institute for Psycholinguistics, PO Box 310, 6500 AH Nijmegen, The Netherlands.

E-mail address: agnieszka.konopka@mpi.nl (A.E. Konopka).
}

regarding information flow across different levels of the production system, in particular regarding the degree to which individual encoding processes unfold independently or in interaction with other processes (see Clifton, Meyer, Wurm, \& Treiman, 2012, for a review of production and comprehension models). For example, theories of lexical access include models that emphasize strictly serial processing of conceptual, lexical and phonological information (e.g., Levelt, Roelofs \& Meyer, 1999; Roelofs, 1997) and 
models that allow interactions between these levels (e.g., Dell, 1986; Dell \& O’Seaghdha, 1992; see Rapp \& Goldrick, 2000, for a review). Theories of structural processing also include models that presuppose a strict separation of structural processes from lexical access (Chang, Dell, \& Bock, 2006; Chang \& Fitz, 2014) and models that allow for interactions between structures and the lexicon (Hartsuiker et al., 2008; Pickering \& Branigan, 1998). Similar questions are found in research on the relationship between syntactic structures and prosodic phrasing (Nakamura, Arai, \& Mazuka, 2012; Tooley, Konopka, \& Watson, 2014; Wagner \& Watson, 2010).

By comparison to lexical, structural and phonological processing, less is known about the processes that put the whole production sequence into motion, i.e., the formulation of the preverbal message (a conceptual representation that captures the speaker's communicative intent) and the mapping of this information onto language (see Konopka \& Brown-Schmidt, 2014, for a review). In general, it is clear that processing at higher levels in the system must constrain processing at lower levels: what speakers want to communicate influences how they communicate it. Thus, for example, conversational history or concerns about audience design can determine the amount of conceptual detail that speakers encode as well as the form of referential expressions (e.g., Arnold, 2010; Brown-Schmidt \& Konopka, 2014). What is unclear is whether and how messages can shape the way speakers formulate, or assemble, sentences expressing their messages online.

This paper aims to identify dependencies between message-level and sentence-level processes during the formulation of simple event descriptions (active sentences like The swimmer is pushing the paparazzo into the pool). While most production theories agree that message and sentence formulation involve a number of processes that unfold incrementally (Levelt, 1989; Wheeldon, 2013), the coordination of these processes is a matter of debate. The approach we take here to identify between-level dependencies is to track the effects of experience-dependent changes in message-level and sentence-level processes on the timecourse of formulation. In two experiments, we manipulate speakers' familiarity with the content of the target messages and with the linguistic structures they can use to express these messages. We then compare effects of message familiarity on formulation against effects of familiarity with the linguistic structures used to express the target messages (Konopka, 2012; Konopka \& Meyer, 2014). Thus we test whether message-level and sentence-level encoding processes influence the timecourse of formulation jointly or independently, and we discuss the implications of these outcomes for understanding the dynamics of processes at the interface of thinking and speaking.

\section{Incrementality and control of formulation}

When preparing to speak, we normally encode sequences of message-level (conceptual) and sentence-level (linguistic) increments. Speakers may, for example, encode a small increment of conceptual information and pass this increment on to linguistic encoding processes before the entire message is complete. At the sentence level, a message increment may then also be encoded linguistically in a sequence of smaller increments. This type of incremental planning leaves room for considerable flexibility in formulation and thus provides a natural test-bed for addressing questions about information flow between levels of the production system.

Studies examining the online formulation of messages and sentences confirm that increment size at both levels can be highly variable. For example, when describing simple objects (e.g., small star), speakers can encode the part of the message that expresses size (small) separately from the part of the message that identifies the referent (star; Brown-Schmidt \& Konopka, 2008, 2014), suggesting that increments can be lexically sized. Similarly, speakers may encode the content of a simple event (The dog chasing the mailman) one character at a time, particularly when one character is more salient or accessible than the other (Gleitman, January, Nappa, \& Trueswell, 2007; Kuchinsky \& Bock, 2010). On the other hand, there is also evidence that message formulation can begin with generation of a larger conceptual representation - i.e., a representation that includes information about relationships between elements of the message (objects or characters) before linguistic encoding begins (Bock, Irwin, \& Davidson, 2004; Griffin \& Bock, 2000; Konopka \& Meyer, 2014; Lee, Brown-Schmidt, \& Watson, 2013).

Comparing across studies, these results provide suggestive evidence that properties of the message may play a key role in controlling formulation. Although the content and structure of messages are difficult to define (see Chang et al., 2006; Konopka \& Brown-Schmidt, 2014), the most striking differences in message properties across studies arguably concern the conceptual cohesiveness of the stimuli used to elicit speech. Notably, speakers appear to encode individual message elements one by one when messages have little internal conceptual structure (e.g., noun phrases describing non-interacting objects; Brown-Schmidt \& Konopka, 2008, 2014; Griffin, 2001; Konopka, 2012; Meyer, Sleiderink, \& Levelt, 1998) or, in the case of events, when the conceptual structure is hard to apprehend (e.g., events where relationships between characters are hard to encode; Kuchinsky \& Bock, 2010). In contrast, speakers appear to prepare larger message increments when a to-be-described event is relatively unambiguous and can be quickly encoded as a conceptually coherent proposition (Konopka \& Meyer, 2014; Kuchinsky \& Bock, 2010). It is therefore possible that differences between messages on this dimension may inherently support different formulation strategies.

The proposal that formulation can vary with the ease of conceptual encoding is consistent with studies showing experimentally-dependent changes in increment size within the same message and sentence types. For example, speakers tend to generate smaller message-level and sentence-level increments under conditions of high cognitive load or time pressure (Ferreira \& Swets, 2002; Wagner, Jescheniak, \& Schriefers, 2010). Increment size also depends on the ease of completing linguistic encoding operations from the outset of formulation: speakers are 
more likely to prioritize encoding of a character in a simple event when this character is easy to identify and to name, and more likely to prioritize encoding information about multiple characters when using a more familiar syntactic structure (Konopka \& Meyer, 2014; also see Konopka, 2012). Fluctuations in the timecourse of formulation that follow from variations in the ease of performing the required encoding operations suggest that formulation is at least partially process-driven. This is also generally consistent with the claim that incrementality is an adaptive feature of the production system, allowing speakers to implicitly choose between formulation strategies that support efficient encoding (see Wheeldon, 2013, for a review; also see Jaeger, 2010, for a discussion of adaptation effects that maximize efficiency in production).

An open question, however, is to what extent formulation varies with ease of conceptual encoding within the same message types. Unlike studies that compared experience-dependent changes in incrementality in the same sentences, between-experiment comparisons provide only indirect evidence of message-level control of formulation. Here, we provide a strong test of this hypothesis by tracking whether the formulation process varies with the ease of encoding the content of a message. In two eye-tracking experiments, we elicit simple event descriptions (e.g., The swimmer is pushing the paparazzo) after exposing speakers to conceptually similar and dissimilar events. Thus, keeping the target message constant across conditions, we examine whether and how familiarity with one event benefits the formulation of a second event description.

A related question is to what extent message-level variables influence sentence-level encoding. If the production system is sensitive to fluctuations in the ease of encoding message-level information, then changes in speakers' familiarity with a message may have consequences for the formulation of the message itself and for deployment of processes that encode the message linguistically. Naturally, similarity in messages often also entails similarity in linguistic expression. For example, messages generated in conversations on any given topic tend to be expressed with a repertoire of conceptually or thematically related words. Likewise, similar messages also tend to be expressed with similar linguistic structures because there are inherent parallels between the conceptual structure of messages and the linguistic structures that can convey this information (see Bock, 1982, for a review of functional accounts of syntax; also see Bunger, Papafragou, \& Trueswell, 2013). ${ }^{1}$ These relationships between message-level and sentence-level structures imply that formulation may be simultaneously constrained by speakers' experience with the message and by the ease of expressing this information linguistically. Thus in both experiments,

\footnotetext{
1 Repetition of words or structures across utterances is also a strategic tool used by speakers for pragmatic or stylistic reasons, and convergence in speakers' lexical or structural choices over time in conversation can be viewed as a form of alignment motivated by communicative concerns (Pickering \& Garrod, 2004). We do not address questions regarding the effects of strategic repetition on formulation here as we use a paradigm where this type of repetition has little communicative value.
}

we also manipulate the ease of generating target descriptions at the sentence level, and we examine to what extent message-level familiarity and sentence-level variables jointly influence formulation.

The approach taken here - i.e., exploiting experience-dependent changes in formulation to identify dependencies between levels of the production system also provides empirical evidence for a larger, ecologically valid question. The current lack of experience-based theories explaining how message familiarity influences formulation is surprising because, of course, language is a communicative tool used to express meaning. Further, the formulation of messages in everyday speech normally implies some degree of experience or familiarity on the part of the speaker with the information that is being communicated: in conversation, similarity in message content across utterances is the rule rather than the exception, and familiarity with message content may increase over time as interlocutors build common ground. This familiarity can then influence both what speakers choose to say and how they express themselves (e.g., Arnold, 2010; Barr, 2008; Brown-Schmidt, 2009; Grice, 1975; Schober \& Clark, 1989; Wilkes-Gibbs \& Clark, 1992). A similar set of constraints may plausibly shape online formulation (also see Von Stutterheim, Flecken, \& Carroll, 2013, for a "thinking for speaking" perspective on these questions).

\section{Current experiments}

Eye-tracked speakers described a long series of pictured events, including target pictures showing two-character events (events with an agent and a patient; Fig. 1) that elicited active and passive descriptions in Dutch. Both experiments used a two-factor prime-target paradigm. First, speakers' familiarity with the content of the target events was manipulated by presenting conceptually similar or dissimilar events on the preceding prime trial (event primes). The aspect of message content that we focus on is relational information. In light of studies showing strong effects of relational processes on formulation (Kuchinsky \& Bock, 2010), we varied the similarity in the relationships between event characters in the prime and target events: prime pictures showed two characters engaged in an action that was conceptually similar or dissimilar to the action shown in target events. For example, the target event showing a swimmer pushing a paparazzo was presented after a prime picture showing a hippie tripping a waiter (similar action) or a hippie paying a waiter (dissimilar action). Second, the ease of expressing relational information at the sentence level was manipulated orthogonally by presenting the prime events with pre-recorded active or passive descriptions (structural primes; Konopka \& Meyer, 2014).

Given the centrality of relational information in the manipulations we used to influence the ease of message-level and sentence-level encoding, we compared the effects of these manipulations on the formulation of sentences with different verb placement. Verbs, by definition, express relationships between elements of a message (in this case, relationships between characters in an event). In Experiment 1, speakers generated subject- or 


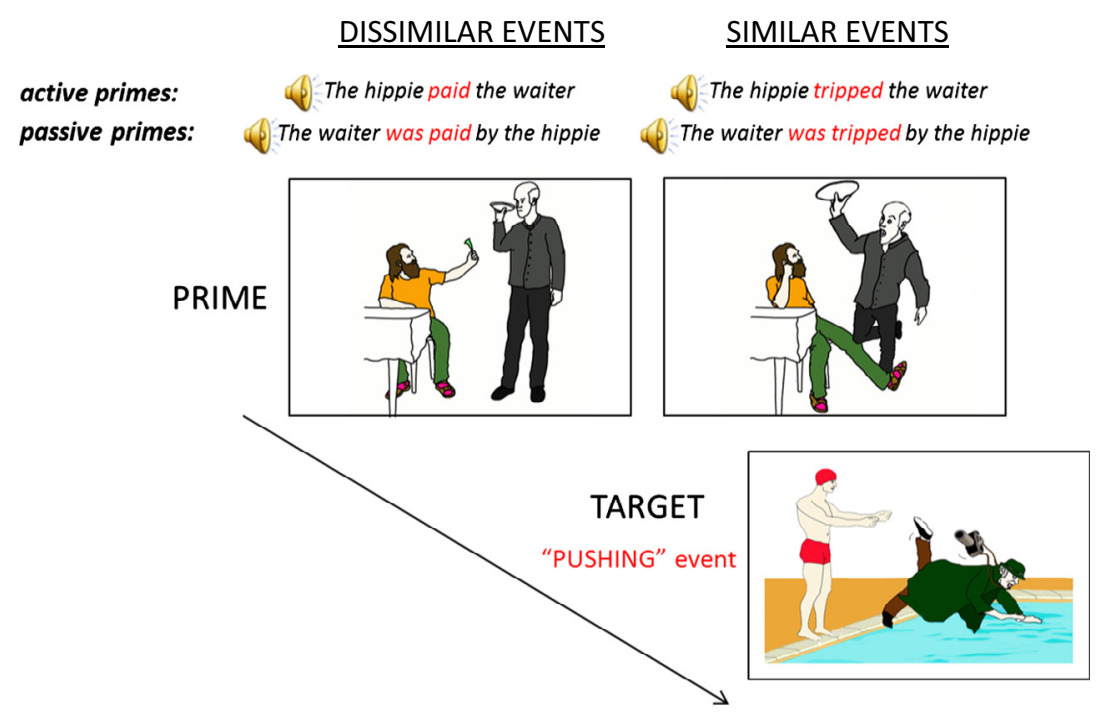

Fig. 1. Example of a prime-target event pair across conditions. Speakers described target events after seeing conceptually similar or dissimilar prime events (event primes) accompanied by recorded active or passive descriptions (structural primes). In Experiment 2, a prompt word was presented before the onset of target pictures.

object-initial sentences (sentences with canonical verb-medial SVO/OVS structures, as in English), and in Experiment 2, they were prompted to produce verb-initial sentences. Production of the verb before its arguments is required in Dutch main clauses when sentences begin with adjuncts (e.g., Vandaag... [Today...]). Compared to SVO sentences, active sentences change only the order of verbs and nouns (Vandaag duwt de zwemmer de paparazzo; the equivalent of [Today] [is pushing] [the swimmer] [the paparazzo] in English), and thus have VSO word order. Passive sentences separate the verb's auxiliary and the main verb (Vandaag wordt de paparazzo geduwd door de zwemmer; [Today] [is] [the paparazzo] [pushed] [by the swimmer]) and thus use aux-OVS syntax. Since the adjunct does not add significant content to the sentence and since the verb is the first content word in VSO/aux-OVS descriptions, the sentence completions in Experiment 2 will be referred to as verb-initial sentences for clarity.

In both experiments, we first verified whether speakers were sensitive to prime-target similarity by comparing repetition of structure from conceptually similar and dissimilar prime sentences to target sentences. Speakers reliably repeat sentence structures across conceptually unrelated sentences (structural priming; Bock, 1986), but priming is normally stronger when sentences also repeat content words or use semantically and phonologically related words (the lexical boost, semantic boost, and phonological boost in structural priming respectively; Cleland \& Pickering, 2003; Pickering \& Branigan, 1998; Santestban, Pickering, \& McLean, 2010). In the current experiments, if the similarity of prime and target events is sufficiently strong to influence production, speakers should repeat the structure of prime sentences more often when describing conceptually similar than dissimilar target events (also see Bunger et al., 2013).

We then test how the event primes and structural primes influence the timecourse of formulation of active sentences (i.e., sentences with the preferred linguistic structure). Compared to the conceptually dissimilar event primes, the similar event primes were expected to facilitate early encoding the relationship between characters in the target events and thus support early generation of a conceptual framework for the message. The structural primes were expected to support early generation of a linguistic framework for the target sentences. The structural primes provide the sentence-level analogue of the message-level manipulation of event similarity (linguistic structures support expression of relationships between event characters), so the two types of primes were expected to result in qualitatively the same types of changes in the timecourse of formulation of target sentences.

As in earlier studies using this methodology, we tracked participants' eye movements to examine how they scanned events in preparation for speaking. The first $400 \mathrm{~ms}$ of picture viewing are assumed to involve a combination of conceptual and linguistic encoding. Speakers normally begin fixating one of the two characters preferentially (the subject) by $400 \mathrm{~ms}$; however, fast shifts of gaze to the subject show priority encoding of this character (Gleitman et al., 2007; Kuchinsky \& Bock, 2010), while slower divergence of fixations to the two characters is more consistent with speakers fixating both characters in order to first encode information about the event as a whole and thus generating a conceptual framework for the entire message (Griffin \& Bock, 2000; Konopka \& Meyer, 2014). Linguistic encoding is assumed to start when fixations to the two characters diverge (either before or after $400 \mathrm{~ms}$ ). Once fixations diverge, speakers generally tend to maintain their gaze on the fixated character (the subject) until they retrieve a suitable name and then, around speech onset, they shift their attention to the second character (the object; i.e., these are name-related gazes; Griffin, 2004).

How does familiarity with conceptual information (as manipulated by event primes) shape formulation? We 
outline two different accounts of coordination between message-level and sentence-level processes. The first possibility is that familiarity with a message has little effect on formulation. In principle, there may be different constraints on incremental planning at the message level and at the sentence level: messages can be generated as unstructured representations, but sentence-level encoding must respect linear word order. At the extreme, it is therefore possible that the timecourse of formulation within the same message type is controlled only by linguistic variables: formulation may depend on variables that directly influence speakers' ability to assemble words in the required linear order (sentence-level encoding), but may be relatively insensitive to variables that influence generation of the preverbal message itself (message-level encoding). In other words, there may be little room for variability driven by message properties during the formulation of sentences with a fixed word order. We refer to this option as Linguistic control of formulation.

The alternative is that formulation is sensitive to variations in message content, and therefore that manipulating the ease of message-level encoding can influence both message-level and sentence-level processing. This type of Joint control of formulation may be observed at two points in time. On the one hand, differences in familiarity with an event may influence early formulation: familiarity with an event may facilitate encoding of a larger conceptual framework for the target event (a pushing event with a pusher and pushee; Griffin \& Bock, 2000), while lack of familiarity may result in encoding of a much smaller increment (an increment consisting of information about only one character with an unspecified thematic role, e.g., the swimmer; Gleitman et al., 2007). As a result, differences in eye movements should be observed as early as 0400 ms: speakers should be more likely to distribute their gaze between agents and patients when describing familiar events than less familiar events. We refer to this option as Early Interactivity (i.e., conceptually-supported early formulation). On the other hand, effects of message familiarity may emerge only once speakers begin linguistic encoding. On Griffin and Bock's (2000) account, the conceptual framework for the message generated immediately after picture onset (pre-400 ms) supports deployment of attention to event characters in the order of mention during linguistic encoding (post- $400 \mathrm{~ms}$ ). Thus if familiarity with a message facilitates generation of such a framework, effects of event familiarity may be observable only once this framework is in place to guide further encoding: familiarity with an event may support more consistent, goal-driven deployment of attention to event characters after $400 \mathrm{~ms}$, lasting until the completion of articulation. We refer to this option as Late Interactivity (i.e., conceptually-supported linguistic formulation).

Importantly, we test whether familiarity with a message interacts with availability of the syntactic structures used to express this message: how do event primes and structural primes shape formulation? The accounts outlined above make different predictions with regard to the effects of sentence-level variables on formulation. Linguistic control of formulation predicts that formulation should be sensitive only to variations in the ease of linguistic encoding. Replicating Konopka and Meyer (2014), fast generation of a syntactic structure should facilitate relational encoding: selecting a primed structure should favor early planning of information about both characters $(0-400 \mathrm{~ms})$, whereas selecting a different structure may limit early planning to the first character. In contrast, Joint control of formulation predicts effects of both message-level and sentence-level variables on formulation. On the one hand, the event primes and structural primes may influence eye movements during formulation independently, resulting in additive effects of message-level and sentence-level processes on formulation. On the other hand, message similarity may interact with - and thus either increase or reduce - any effects of structural primes on formulation. One possibility is that event descriptions will be easiest to formulate when speakers are familiar with the target event and when they can repeat a recently experienced syntactic structure: this would predict more structural priming across conceptually similar than dissimilar prime-target event pairs. A second possibility is that message-level facilitation may have a stronger effect on formulation than sentence-level facilitation, and thus that event familiarity will reduce the influence of the structural primes on eye movements. The extent to which such effects occur earlier or later during formulation should distinguish between the Early and Late Interactivity accounts.

In all cases, larger effects of the event primes should be observed in Experiment 2, where verbs are produced sentence-initially, than in Experiment 1, where verbs were produced sentence-medially. Formulation theories that propose early encoding of a conceptual framework for the entire event (e.g., Griffin \& Bock, 2000) predict that speakers should encode relational information in the earliest stages of formulation regardless of word order. However, cross-linguistic comparisons show that speakers prioritize encoding of message elements and content words roughly in the order required by the grammar of the target language (Hwang \& Kaiser, 2014; Norcliffe, Konopka, Brown, \& Levinson, 2015; Sauppe, Norcliffe, Konopka, van Valin, \& Levinson, 2013). This implies that verb placement in transitive sentences should influence the timing of relational encoding, such that formulation may be more sensitive to the similarity in the prime-target event actions when speakers have to produce the verb at the beginning of the sentence.

\section{Overview of analyses}

Experiments 1 and 2 track, for the first time, the formulation of active verb-medial and verb-initial sentences (SVO vs. VSO) with the same message-level content after exposure to event primes (manipulating message-level familiarity) and structure primes (manipulating sentence-level familiarity). Effects of the primes were expected on the surface form of target descriptions and on the timecourse of formulation, as outlined below.

\section{Sentence form}

Speakers were expected to repeat the structure of the primes more often when describing conceptually similar 
than dissimilar events, producing a semantic boost in structural priming. So far, a semantic boost has been reported only for noun phrases (e.g., priming between phrases like the sheep that's red and the goat that's red). Enhancement of priming with conceptual overlap in content words is assumed to result from co-activation of related conceptual nodes (sheep, goat) and their feed-forward effects on lemma selection and structural assembly (Cleland \& Pickering, 2003). For verbs, a semantic boost would demonstrate activation of conceptually similar verbs (tripping, pushing), and thus activation of similar relational information at the message level. An open question is whether a semantic boost will be observed in both verb-medial and verb-initial sentences.

\section{Formulation}

Are observable changes in sentence form after the two types of primes accompanied by changes in formulation? We report analyses that took into account (1) the link between early gaze shifts and sentence form, and (2) changes in the entire distribution of fixations to event characters across conditions before speech onset.

1. First fixations. Speech must start somewhere: at picture onset, speakers can direct their attention to any character in an event and can choose to begin their sentences with either character. On different accounts, formulation may begin with speakers prioritizing encoding of the character that is fixated first (Gleitman et al., 2007) or with speakers distributing their attention between two characters before selecting one of them as the sentence subject (Bock et al., 2004; Griffin \& Bock, 2000). The influence of first fixations on sentence form thus provides a first, coarse-grained measure of formulation strategies.

Kuchinsky and Bock (2010) showed that the relationship between first fixations and sentence form is modulated by event properties: in their experiments, speakers were less likely to assign first-fixated characters to subject position in events that were easy to describe (higher-codability events) than in events that were harder to describe (lower-codability events). Thus when speakers can quickly encode relational information in an event, selection of a starting point is less biased by early availability of information about a single character. We verify whether the codability of events used in these experiments has a similar effect on the relationship between first fixations and sentence form. We also apply the same logic to our manipulation of event similarity. Priming of message content with event primes may facilitate generation of a message-level framework in the same way that high event codability increases early availability of this framework: consequently, speakers should be less likely to begin sentences with the first-fixated character in target events after receiving conceptually similar than dissimilar event primes.

2. Timecourse of formulation. We focus on fixations in two theoretically relevant time windows: fixations between $0 \mathrm{~ms}$ and $400 \mathrm{~ms}$, and fixations between $400 \mathrm{~ms}$ and the average time of gaze shifts from the subject to the object character. If event similarity does not influence the timecourse of formulation (Linguistic control of formulation), there should be no difference in the distribution of fixations to target characters after conceptually similar and dissimilar prime events either before or after $400 \mathrm{~ms}$ However, if processing of one event does facilitate encoding of a similar event, there should be evidence of conceptual guidance in either the early or late time window. Changes in the distribution of fixations occurring before $400 \mathrm{~ms}$ would be strong evidence of message-level control from the outset of formulation (Early interactivity): exposure to a similar prime event should result in slower divergence of fixations to agents and patients in target events, indicating an early preference for encoding the event as a whole. Changes observed only after $400 \mathrm{~ms}$ would indicate message-level control of the deployment of attention during linguistic encoding (Late Interactivity): speakers should fixate the subject character more consistently after receiving conceptually similar than dissimilar event primes.

Analogous predictions apply to the formulation of structurally primed and unprimed sentences. Eye movements should show evidence of linguistic guidance. Before or around $400 \mathrm{~ms}$, speakers should be more likely to distribute their gaze between two characters when repeating the structure of the prime sentences, indicating earlier relational encoding than when using a different structure (Konopka \& Meyer, 2014). After $400 \mathrm{~ms}$, the availability of a linguistic framework should support goal-driven deployment of attention to the subject character, resulting in more consistent fixations in structurally primed than unprimed sentences. The key comparisons in both experiments concern the effects of structural primes on the formulation of target events that are conceptually similar or dissimilar to the primes.

\section{Experiment 1}

Experiment 1 elicited SVO/OVS event descriptions.

\section{Method}

\section{Participants}

64 native speakers of Dutch (students at Radboud University Nijmegen) participated for payment. Eight were replaced because of low numbers of scorable target sentences or technical problems.

\section{Materials and design}

Target pictures showed two-character events $(n=36)$. There were 29 items with animate agents ( 22 humans, 7 animals), and 7 with inanimate agents. 27 of these items had animate patients (all human) and 9 had inanimate patients. $^{2}$

The experiment used a within-participant and within-item 2 (Event Similarity) $\times 2$ (Prime Structure) design (Fig. 1). Target trials were preceded by prime trials where speakers saw pictures of events with two novel

\footnotetext{
${ }^{2}$ Character animacy had the expected effect on sentence form in both experiments and is thus not discussed further: speakers produced a greater proportion of active sentences to describe events with human than non-human agents (.75 vs. .41 in both experiments). Structural priming was observed primarily in events with human agents.
} 
characters and heard recorded descriptions. In the dissimilar event condition, the action shown in the prime event was conceptually unrelated (e.g., paying) to the action shown in the following target picture (e.g., pushing), and in the similar event condition, the prime action was conceptually related (e.g., tripping) to the action in the target picture. The verb used to describe the prime event in the similar event condition was not suitable for describing the target event, ensuring that the content of speakers' descriptions of the target event did not differ across conditions. Pictures were accompanied by active or passive descriptions recorded by a native Dutch speaker.

The placement and configuration of the agent and patient characters in the conceptually similar and dissimilar versions of each prime picture were matched as closely as possible, reducing the potential for lower-level perceptual correlates of event similarity in the similar prime-target event pairs (see Hafri, Papafragou, \& Trueswell, 2013). In addition, two mirror-reversed versions of each prime and target picture were created to counterbalance the left and right hand-side placement of the agent on the screen. Thus there were two prime-target pairings where the placement of agents in prime and target pictures was the same (both characters appearing on the left- vs. right-hand side) and two in which it was different. Crossing this manipulation with the Event Similarity and Prime Structure variables resulted in sixteen lists of stimuli, with each target picture being presented in a different Event Similarity condition, Prime Structure condition, and with a different spatial layout of characters on each list (analyses collapsed across the last factor). Within lists, there were no two prime-target pairs from the same condition presented back to back.

Prime-target pairs were separated by four to five intervening filler trials and word trials. Filler trials showed unrelated events eliciting mostly intransitive sentences: speakers either described the events themselves $(n=106)$ or heard recorded descriptions and repeated them aloud $(n=14)$. On word trials, participants saw words that had been used previously in the experiment (e.g., names of characters from earlier pictures; $n=18$ ) or novel words $(n=15)$ printed in the center of the screen. The word trials were included to support a cover story that participants' memory for sentences would be tested at random intervals.

\section{Picture and sentence norming}

The prime-target picture pairs were normed for similarity in three studies ( $n=46$ participants per study). All participants saw 72 slides with two pictures each. Half of the slides showed pairs of unrelated filler pictures, and half showed prime-target picture pairs. Eight picture lists were created to counterbalance prime-target event similarity (conceptually similar vs. dissimilar pairings), character orientation (left- vs. right-hand side agent placement), and the match in agent orientation in primes and targets (matching vs. mismatching agent orientation).

In the first study, the pictures were presented without descriptions (no-context); in the second study, an active sentence was printed above the first picture (one-sentence context); and in the third study, both pictures were presented with a printed active description (two-sentence context). For prime-target pairs, the sentence printed above the first picture was the recorded prime sentence presented during the main experiment; the sentence printed above the second picture was the modal description of the corresponding target event (determined on the basis of earlier studies; e.g., Konopka \& Meyer, 2014). Participants were instructed to rate the similarity of the action shown in the two pictures on a 5 -point scale ( 1 = very dissimilar; $5=$ very similar).

The purpose of the first study (no-context) was to verify whether participants recognized the conceptual similarity of prime-target event pairs based on their own interpretations of the pictures and in the absence of linguistic cues. The second study (one-sentence context) tested whether prime-target pairs were judged to be more similar when the prime picture was accompanied by a description (as in the main experiments). The third study (two-sentence context) verified whether speakers' interpretations of similarity in prime-target events were consistent with the expected interpretations.

Fig. 2 shows that participants judged conceptually similar prime-target event pairs to be more related than dissimilar pairs in all studies (all ts $>6$ ). This was confirmed by a main effect of Event Similarity in a 2 (Event Similarity) $\times 3$ (Sentence Context) analysis of variance performed on item means, $F(1,35)=135$. Results from the no-context study suggest that participants easily recognized the similarity in similar prime-target event pairs without any descriptions. Differences in prime-target similarity ratings were larger when pictures were presented with one or two context sentences, resulting in an interaction between Event Similarity and Sentence Context in a separate $2 \times 2$ analysis, $F(1,35)=26.71$. Thus the sentence primes increased participants' perceived similarity of the two events (this may be because the sentences directed attention to the intended interpretation of the event or because of lexical priming). The perceived difference in similarity between conceptually similar and dissimilar prime-target event pairs was larger in the one-sentence than the two-sentence context studies, $F(1,35)=3.71, p=.06$, for the interaction of Event Similarity with Sentence Context in a separate $2 \times 2$ analysis, suggesting that participants' selfgenerated interpretations and judgments of the target events were consistent with the expected interpretations (if anything, speakers rated the pictures as being more similar when they evaluated event similarity in the absence of target descriptions).

To verify whether lower-level, perceptual factors influenced participants' judgments, similarity ratings were also compared across picture pairs with matching and mismatching character orientation: these ratings differed minimally across all three studies (2.64 vs. 2.58 for pictures with matching and mismatching character orientation respectively; a reliable difference was only observed in the one-sentence context study), but did not interact with Event Similarity or Sentence Context, all Fs $<.5$. This confirms that participants' perceptions of event similarity were based primarily on their interpretations of the semantics of the event action. 


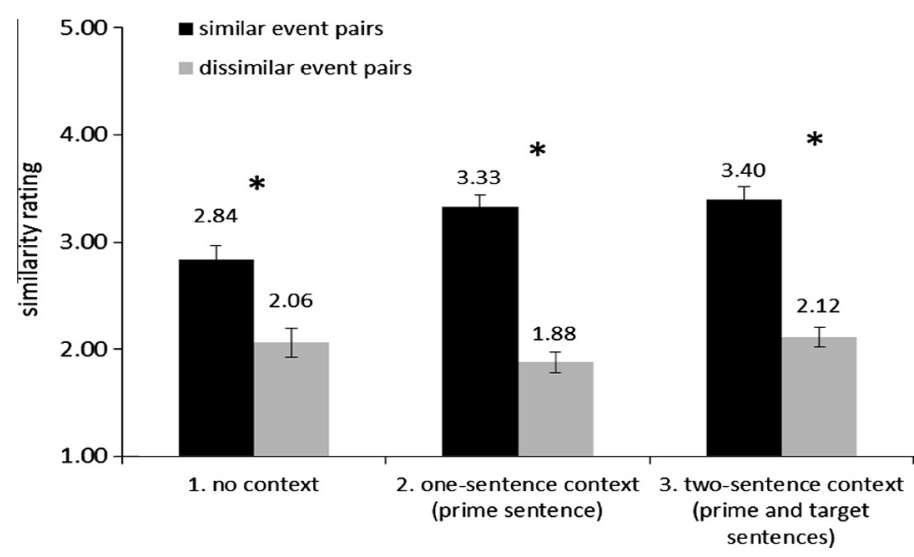

Fig. 2. Mean similarity ratings (with standard errors) for conceptually similar and dissimilar prime-target event pairs in the three norming studies. In each study, the similar and dissimilar event pairs are different at $p<.0001$.

\section{Procedure}

The experiment was carried out with an EyeLink 1000 eye-tracker (1000 Hz sampling rate). We used a 9-point calibration procedure. Instructions appeared on the screen and were paraphrased by the experimenter. Each trial began with a fixation point at the top of the screen. On most trials $(n=142)$, speakers saw pictures of events and were asked to describe them with one sentence, mentioning all characters in the picture ( 36 targets and 106 fillers). On a subset of trials $(n=52)$, participants first saw the word LISTEN, indicating that they would hear a recorded description, and then saw a picture: their task was to listen to the sentence and repeat it aloud (36 primes and 14 fillers). Finally, participants were also told that their memory for content words would be tested throughout the experiment. On a minority of trials $(n=33)$, they saw a printed word instead of a picture: their task was to read this word out loud and say "yes" or "no" to indicate whether they had already used or heard this word in the experiment.

\section{Sentence scoring}

Target sentences were scored as actives, full passives, or truncated passives (The swimmer is pushing the paparazzo; The paparazzo is being pushed by the swimmer; The paparazzo is being pushed ${ }^{3}$ ). Sentences with other constructions were rejected. 32 additional sentences were excluded because speakers repeated the prime verb in target sentences ( $1.7 \%$ of all scored trials). Trials were also excluded if the first fixation in that trial fell on one of the characters in the picture rather than the central fixation point, and if onsets were longer than $5 \mathrm{~s}$ and 2.5 standard deviations from the overall mean. The final dataset consisted of 1529 sentences (61\% actives, $27 \%$ full passives, $12 \%$ truncated passives).

\footnotetext{
${ }^{3}$ Dutch translations: De zwemmer duwd de paparazzo; De paparazzo wordt geduwd door de zwemmer; De paparazzo wordt geduwd.
}

\section{Event and character codability scoring}

Event Codability was estimated with Shannon's entropy (Kuchinsky, 2009): events consistently described with a small set of verbs received high Event Codability scores and events eliciting a broader range of verbs received lower Event Codability scores. Higher-codability events are considered to be easier to apprehend and describe, while lower-codability events are harder to describe. The ease of encoding agents and patients (Agent and Patient Codability) was estimated with the same procedure on the basis of heterogeneity in character names; we include these variables for control purposes and report their effects briefly for completeness.

All three codability indices showed large between-item differences but were not inter-correlated (all $r s<.22, n s$ ). Event codability did not differ in target sentences produced after conceptually similar and dissimilar prime events, $t(34)=.54$, showing that the primes had no discernible influence on speakers' interpretations of the target action and their verb choices. This validates our manipulation as influencing the way that speakers might formulate event descriptions rather than changing the content of the message itself. Event Codability was also not related to the similarity ratings from the one-context norming study.

\section{Analyses}

Analyses of structure choice and speech onsets were conducted with mixed logit models and linear mixed effects models in $\mathrm{R}$, including participants and events as random effects (Baayen, Davidson, \& Bates, 2008; Jaeger, 2008). All models included a combination of the following predictors: Event Similarity, Prime Structure, and the location of First Fixations as categorical predictors, as well as Event Codability, Agent Codability, and Patient Codability as continuous predictors. Prime-target Similarity ratings (from the no-context and one-sentence context norming studies) were included in separate models as an alternative to the binary predictor of Event Similarity. All predictors 
were centered. We report results from models that included the highest-level interactions of interest and the full random structure leading to convergence (Barr, Levy, Scheepers, \& Tily, 2013). Effects were considered to be reliable at $p<.05$.

Timecourse data were subjected to Growth Curve Analysis (GCA; Mirman, 2014; Mirman, Dixon, \& Magnuson, 2008). Analyses were performed on the proportions of agent-directed fixations (agent and patient fixations are largely complementary). GCA is a type of multilevel regression that has been increasingly applied to eye-tracking studies to analyze nonlinear changes in the proportion of fixations over time. Orthogonal polynomial terms are fit to fixation data to describe its shape. Beta values $(\beta)$ describe the fit of each parameter, such as the mean proportion of fixations (intercept term) and the slope of fixations across a time window (linear term). Larger intercept betas indicate an overall greater mean proportion of agent fixations, while larger positive slope values indicate a larger proportion of agent-directed fixations in the later than the earlier part of the time window (i.e., more sustained or increasing looks to the agent).

Higher-order polynomials are included to capture inflection points (non-linearities) in the pattern of fixations. The shape of the primary inflection is described by a quadratic term, with positive betas indicating a more linear, flatter (stable across time) pattern of agent fixations, and negative betas indicating a greater peak (larger fluctuations) in fixations. A secondary inflection is captured by the cubic term. Positive cubic betas generally push the fit of the primary peak earlier and to occur more transiently (steep rise and fall). Negative betas pull the fit of the primary peak later and to be more sustained (e.g., Kalénine, Mirman, \& Buxbaum, 2012; Kalénine, Mirman, Middleton, \& Buxbaum, 2012; Kuchinsky et al., 2013; Mirman et al., 2008). ${ }^{4}$

GCA was implemented using R (R Core Team, 2014) and the lme4 package (Bates, Maechler, Bolker, \& Walker, 2014). Fixed effects (polynomial and condition regressors) and random effects (error terms) were modeled to predict the proportions of agent fixations. To maximize random effects, random intercepts and slopes were included for the polynomials as well as interactions among the experimental conditions.

Contrast-coded fixed effects in the analyses included interactions between the two manipulations of interest (Event Similarity, Prime Structure) with orthogonal polynomials (intercept, linear, quadratic, and cubic terms). Separate by-participant and by-item models were run with the maximal random structures. Effects were considered to be reliable when their removal resulted in significantly poorer model fit, $\chi^{2}$ test $p<.05$.

\footnotetext{
4 Additional higher-order polynomials (e.g., quartic terms) can be added, though with decreasing theoretical interpretability (higher-order interactions generally fit the tails of the distribution) and increased likelihood of overfitting. Such polynomials do not alter the estimates of lower-order effects given that they are orthogonalized. Because of these concerns, analyses included only intercept through cubic terms, which provided a good fit for the data.
}

\section{Results}

\section{Semantic boost in structural priming}

Speakers' choice of sentence structure on target trials (actives vs. full passives) showed a strong semantic boost (Fig. 3a): target descriptions repeated the structure of prime sentence more often in conceptually similar than dissimilar prime-target event pairs (11\% vs. $2 \%$ priming effects), resulting in an interaction of Event Similarity with Prime Structure $(\beta=.74, z=2.08)$.

Two complementary analyses were carried out using the Prime-target Similarity ratings obtained for each prime-target pair in the no-context and the one-sentence context norming studies (see Fig. $3 \mathrm{~b}$ for the latter) instead of the binary variable of Event Similarity. Both analyses showed that the magnitude of the semantic boost increased with increasing similarity in the prime and target events ( $z$ s $>2.5$ for the interaction of Prime Structure with the two types of similarity ratings).

\section{Production of full passives and truncated passives}

Due to high rates of production of two types of passives (full passives and truncated passives), a separate analysis tested whether rates of agent mention also varied across conditions. Overall, speakers produced more full passives (conversely, fewer truncated passives) after receiving passive primes than active primes. Importantly, full passives were produced at comparable rates after conceptually dissimilar passive and active event primes (.67 vs. .68), but were produced more often after conceptually similar passive primes than similar active primes (.77 vs. .60). This resulted in an interaction of Event Similarity with Prime Structure, $\beta=3.99, z=3.87$ ( $z s=1.38$, ns, and 3.32 for the same interaction in models run with the two types of Prime-target Similarity ratings). Thus exposure to a similar event described with passive syntax supported generation of a two-slot passive frame for target descriptions (one patient slot and one agent slot) and filling of both slots in this frame.

\section{Priming effects across different event types}

Separate analyses tested whether priming effects varied with properties of the events. As prime-target similarity concerned similarity in the event action, we focus on interactions between Event Similarity, Prime Structure and Event Codability.

Speakers repeated the structure of the primes more often when describing higher-codability than lower-codability target events (priming effects of .11 vs. .04 respectively after a median split of items into higherand lower-codability events; Fig. 4a). This resulted in an interaction of Event Codability with Prime Structure (all $z s>2.6$, in models run with Event similarity as a categorical predictor as well as models with Prime-target Similarity ratings as continuous predictors). Thus exposure to an active or passive prime sentence had a stronger effect on the form of target sentences when the target event was easy to apprehend and describe (high codability) than 
(a) Experiment 1

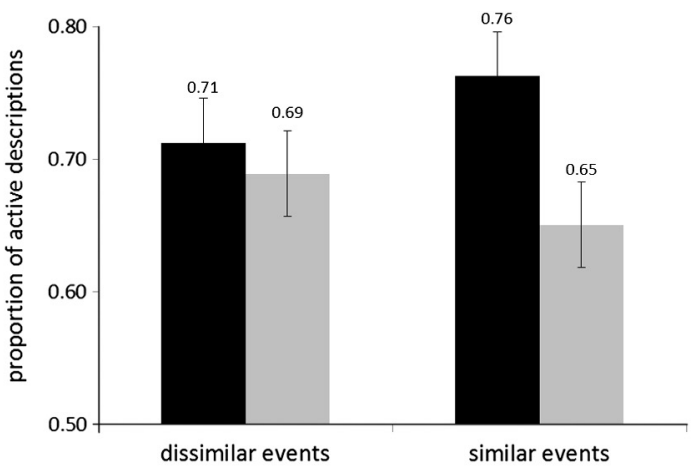

(b) Experiment 1

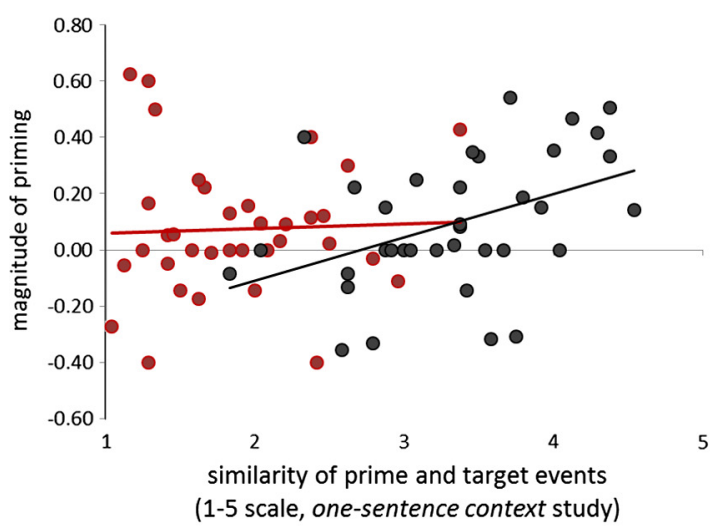

(c) Experiment 2

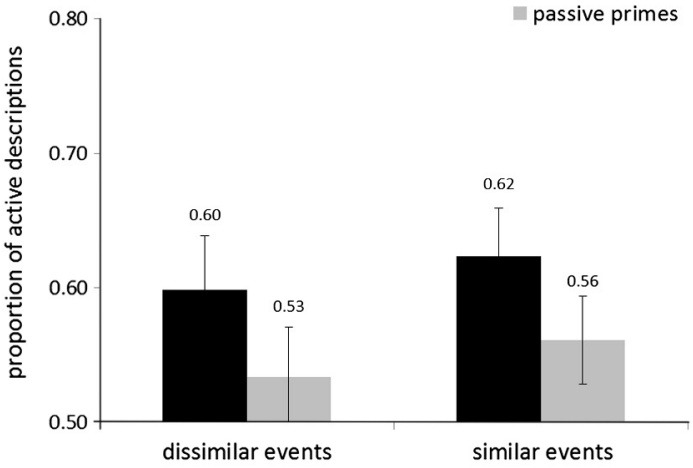

(d) Experiment 2

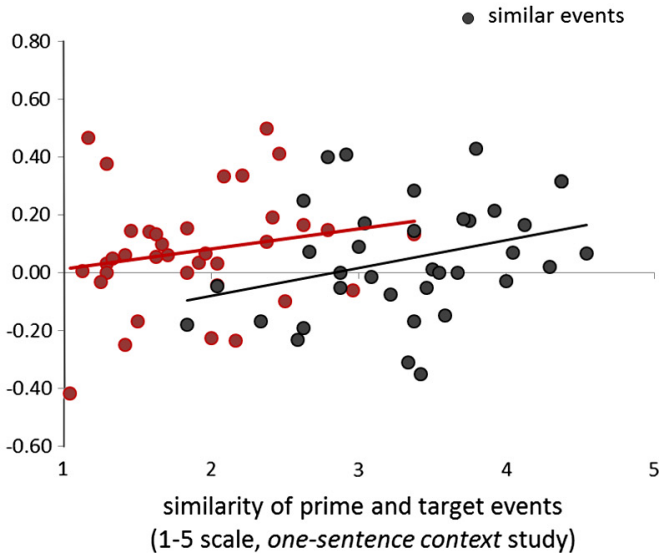

Fig. 3. Proportions of active sentences produced on target trials in ( $a$ and $b$ ) Experiment 1 and ( $c$ and d) Experiment 2 as a function of the event primes and structural primes (with standard errors). Figures ( $b$ and d) show the magnitude of structural priming by item with respect to prime-target event similarity ratings obtained in the one-sentence context norming study.

(a) Experiment 1

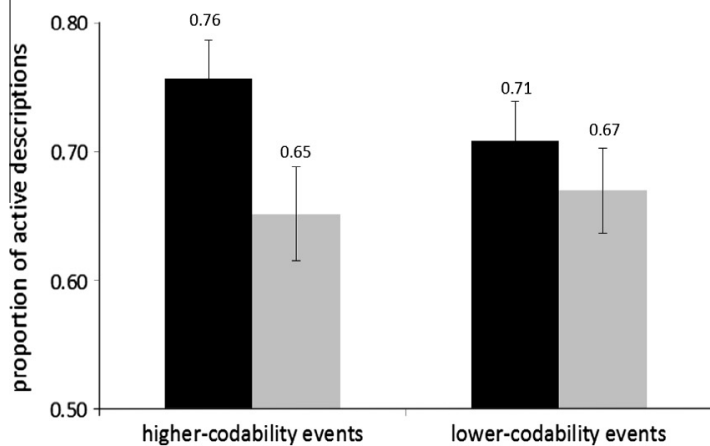

(b) Experiment 2

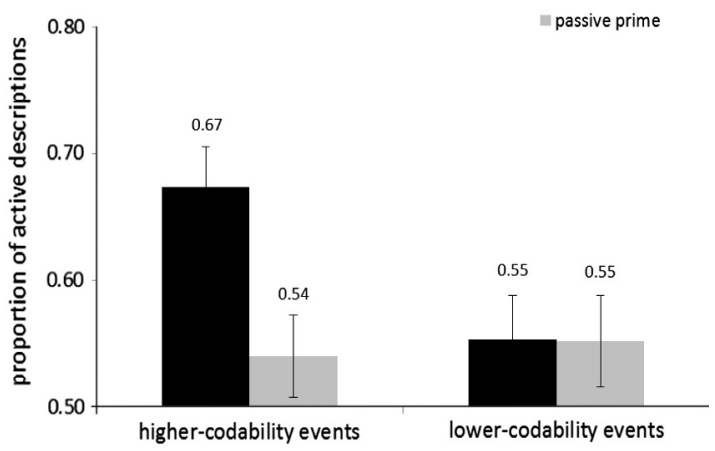

Fig. 4. Proportions of active sentences describing higher-codability and lower-codability events after active and passive primes (with standard errors) in Experiments 1 and 2 .

when it was more difficult to apprehend (low codability). This suggests that transfer of a linguistic structure from one sentence to another depended, in part, on the ease of encoding the conceptual structure of the target event. In other words, fast generation of a conceptual framework for the message and a linguistic framework for the sentence may facilitate the message-to-language mapping. The three-way interaction of Prime Structure, Event Similarity and Event Codability was not reliable in any of the three analyses (all $z \mathrm{~s}<.9$ ). 
Effects of Agent and Patient Codability on sentence form were considered separately. Consistent with earlier demonstrations of accessibility effects on sentence form, speakers produced more actives to describe events with easier-to-name than harder-to-name agents (.72 vs. .66) and more passives to describe events with easier-to-name than harder-to-name patients (.65 vs. .74). However, Agent Codability interacted only weakly with Prime Structure $(z=1.58, n s)$ and effects of Patient Codability did not reach significance.

\section{Speech onsets}

Speech onsets did not differ across active and passive sentences (2058 vs. $2100 \mathrm{~ms}$ ), and were compared for each sentence type individually with respect to Prime Structure, Event Similarity, Event Codability, and Agent or Patient Codability. Analyses showed that onsets were sensitive only to properties of agents and patients when these characters were produced in subject position: active sentences were initiated more quickly when events included easy-to-name than harder-to-name agents ( $\beta=116$, $t=2.29$, for the main effect of Agent Codability), and passive sentences were initiated more quickly when events included easy-to-name than harder-to-name patients ( $\beta=190, t=2.94$, for the main effect of Patient Codability). Thus before speech onset, speakers had lexically encoded only the first-mentioned character.

\section{Timecourse of formulation}

Timecourse analyses tested whether and how the formulation of target sentences was influenced by familiarity with the message content (event primes) and by the ease of generating linguistic structures (structural primes).

\section{First fixations and sentence form}

Speakers directed a greater proportion of first fixations to agents than patients (.58 vs. .42), but the distribution of first fixations did not differ across conditions. Importantly, speakers were more likely to produce active sentences when they first fixated agents than patients (.71 vs. .67).

(a) higher-codability events

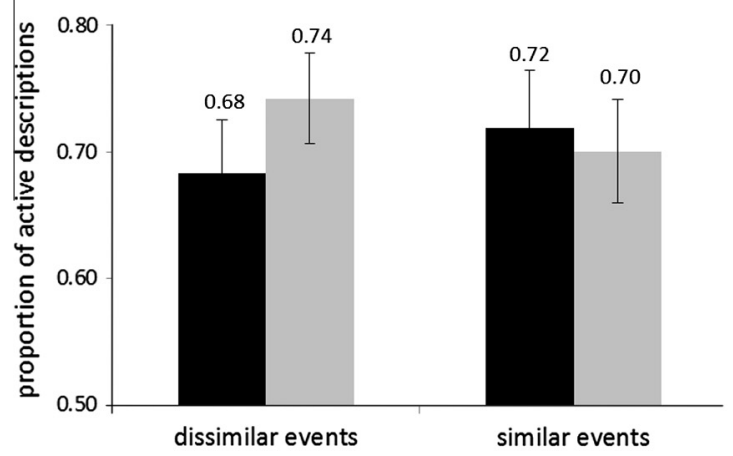

A model including First Fixations, Prime Structure, Event Similarity and Event Codability showed that the effect of First Fixations on sentence form varied with both Event Codability and Event Similarity. First Fixations had little to no influence on sentence form in higher-codability events (Fig. 5a), but were better predictors of sentence form in lower-codability events (Fig. 5b), particularly when prime and target events were conceptually dissimilar $(z \mathrm{~s}=2.9,1.4$ and $1.4[n s]$, for the three-way interaction of these factors in models run with Event Similarity and the two types of Prime-target Similarity ratings respectively; the magnitude of the interactions was reduced by random slopes).

The results are consistent with Kuchinsky and Bock's (2010) observation that early attention shifts did not uniformly predict assignment of characters to subject position. In particular, first fixations did not predict sentence form when speakers can rely on conceptual information to select a suitable starting point - such as when describing higher-codability events or, as shown here, after exposure to conceptually similar prime events. In contrast, speakers were more likely to fall back on a simple strategy of assigning a first-fixated character to subject position when a message-level framework was harder to generate - such as when events are hard to encode (lower-codability events) or when the event content is less familiar (target events that are conceptually dissimilar to prime events).

\section{Timecourse}

The timecourse of formulation was assessed only for active sentences (due to sparse data for passives). Fixations to agents and patients were binned into consecutive $10 \mathrm{~ms}$ samples, and the proportion of agent-directed fixations in each sample was calculated per participant and per item over the total number of fixations in that sample (fixations to the agent, the patient, and fixations falling outside of these areas).

Analyses were performed on agent-directed fixations in two time windows: early $(0-400 \mathrm{~ms})$ and late (400$1500 \mathrm{~ms}$ ). The first window includes a period of minor gaze shifts between agents and patients, while the second (b) lower-codability events

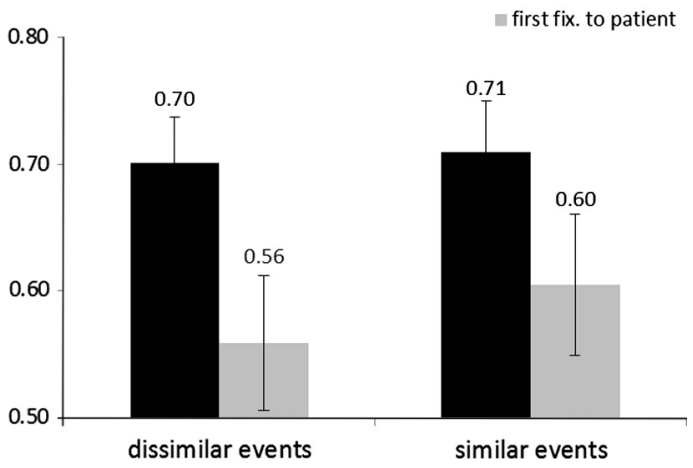

Fig. 5. Proportions of active sentences in Experiment 1 describing higher-codability and lower-codability events after conceptually similar and dissimilar event primes when first fixations were directed to agents and patients (with standard errors). 
Experiment 1

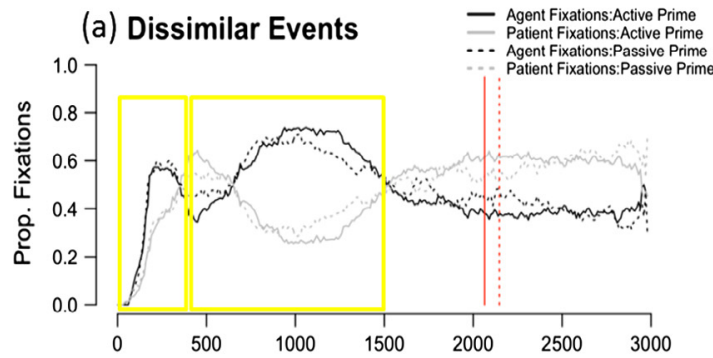

(b) Similar Events

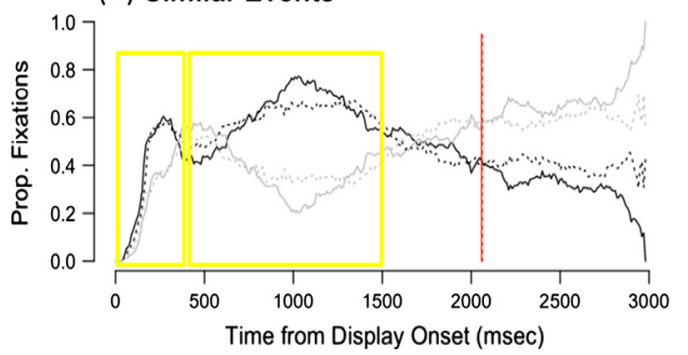

Experiment 2

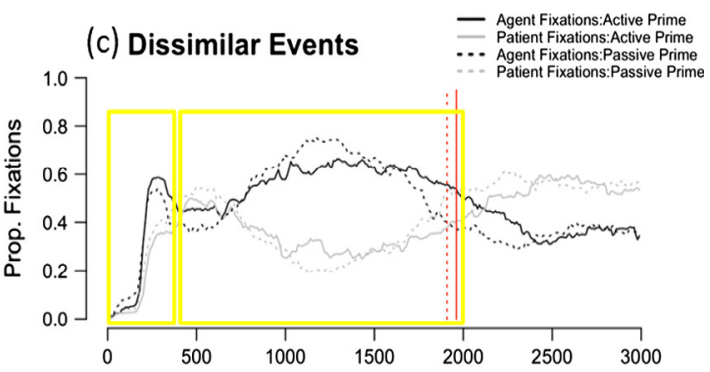

(d) Similar Events

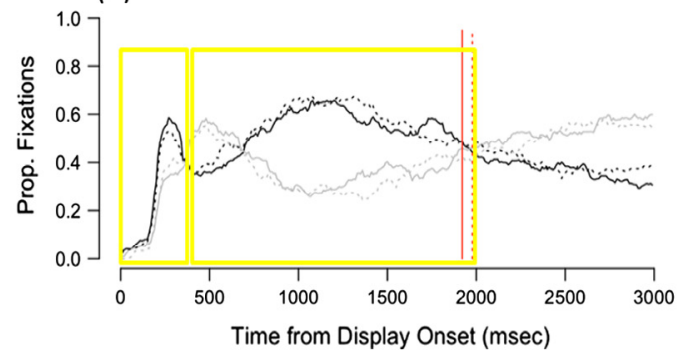

Fig. 6. Formulation of active sentences in (a and b) Experiment 1 (SVO) and (c and d) Experiment 2 (VSO): proportions of agent-directed and patientdirected fixations in dissimilar and similar target events after active and passive primes. Time $0=$ picture onset. Vertical solid and dashed lines indicate speech onsets after active and passive primes respectively. Yellow boxes show the time windows chosen for analysis. (For interpretation of the references to color in this figure legend, the reader is referred to the web version of this article.)

window includes a period of time where speakers deployed their attention preferentially to the agent and maintained fixations on this character before the large gaze shift to the patient at approximately $1500 \mathrm{~ms}$. This partition into early and late time windows closely matches the theoretical distinction between event apprehension (0-400 ms) and linguistic encoding (post-400 ms) outlined by Griffin and Bock (2000). ${ }^{5}$

Descriptively, in all conditions (Fig. 6a and b), speakers first showed a small preference for the agent over the patient (0-200 ms) and then briefly directed their gaze to the patient by $400 \mathrm{~ms}$. After $400 \mathrm{~ms}$, they began fixating the agent preferentially. This pattern of fixations was modulated primarily by Prime Structure and more weakly by Event Similarity.

In the early window (0-400 ms), fixations to the agent were less sustained (negative linear slope; Table 1a) and more peaked (negative quadratic effect, only by-participants) after active primes compared to passive primes. This effect is characterized by the larger drop-off in agent fixations following the peak in the early window in the active prime condition (see Fig. 7a for the raw data and GCA model fits). Interactions of Prime Structure with

\footnotetext{
5 Although it is clear that speakers can apprehend event gist in less than 100 ms (Dobel, Gumnior, Bolte, \& Zwitserlood, 2007; Hafri et al., 2013), the current results show a qualitative difference in fixations pre- and post-400 ms. We use the theory-neutral terms "early" and "late" windows throughout the paper. Performing the same analyses on a wider time window (0-1500 ms) leads to similar conclusions, albeit based on models that include 6th order polynomials to account for multiple inflection points in this larger time window.
}

Event Similarity were inconsistent across the by-participant and by-item analyses (Table $1 \mathrm{~b}$ ).

Following the brief period of fixating patients (200$400 \mathrm{~ms}$ ), agent fixations after active primes rose more slowly in the later window (400-1500 ms). Specifically, fixations to the agent were more sustained (positive linear effect), with a larger peak in fixations (negative quadratic effect) that rose and fell more slowly (negative cubic effect) after active than passive primes (Table 1c). Together, these effects show a slower and more sustained pattern of fixations to the agent during the late window. In other words, speakers fixated agents more consistently and showed a stronger preference for the agent after active primes than passive primes.

Event Similarity had a weaker effect on formulation in this late time window than the structural primes. Interactions of Prime Structure with Event Similarity revealed a smaller effect of structural primes in conceptually similar than dissimilar events (negative linear and negative quadratic terms; Table $1 \mathrm{~d}$ ). To clarify the directionality of the interactions, sub-model tests of the by-subject models examined the effect of the structural primes separately for conceptually dissimilar and similar event prime conditions. The model fits are overlaid for direct comparison in Fig. 7b. All effects listed below were reliable at $p<.0001$.

First, the linear interaction in Table 1d indicates that when describing conceptually dissimilar events, fixations to the agent increased over time more after active primes (solid red lines) than passive primes (dashed red lines; linear $\beta=42.74, t=10.88$ ). This structural priming effect was 
Table 1

Experiment 1: GCA results for active sentences in early and late time windows (0-400 ms, 400-1500 ms). (a and c) Polynomial $\times$ Prime structure: Estimates compare the pattern of agent-directed fixations following active vs. passive primes (the passive prime condition served as the baseline). (b and d) Polynomial $\times$ Prime Structure $\times$ Event similarity: Estimates show how effects of structural primes in (a) differ between similar and dissimilar event conditions (the dissimilar prime condition served as the baseline).

\begin{tabular}{|c|c|c|c|c|c|c|c|c|}
\hline \multirow[b]{2}{*}{ Effect } & \multicolumn{4}{|c|}{ By-participants } & \multicolumn{4}{|l|}{ By-items } \\
\hline & $\beta$ & $t$ & $\chi^{2}$ & $p$ & $\beta$ & $t$ & $\chi^{2}$ & $p$ \\
\hline \multicolumn{9}{|c|}{$\begin{array}{l}\text { Early window }(0-400 \mathrm{~ms}) \\
\text { (a) Prime structure (Active }>\text { Passive) }\end{array}$} \\
\hline Intercept & -.12 & -.06 & .004 & .95 & -.76 & -.41 & .17 & .68 \\
\hline Linear & -11.59 & -3.77 & 14.23 & $<.001$ & -7.59 & -2.90 & 8.42 & .004 \\
\hline Quadratic & -9.52 & -3.07 & 9.43 & .002 & -2.89 & -1.11 & 1.22 & .27 \\
\hline Cubic & 2.36 & .76 & .58 & .45 & .42 & .16 & .03 & .87 \\
\hline \multicolumn{9}{|c|}{ (b) Event similarity (Similar $>$ Dissimilar) $\times$ Prime structure } \\
\hline Intercept & 2.46 & .53 & .28 & .60 & -.09 & -.03 & .001 & .98 \\
\hline Linear & -2.43 & -.45 & .21 & .65 & -.39 & -.08 & .007 & .93 \\
\hline Quadratic & -6.66 & -1.23 & 1.51 & .22 & 9.58 & 2.00 & 3.99 & .05 \\
\hline Cubic & 3.17 & .59 & .35 & .56 & 6.10 & 1.27 & 1.62 & .20 \\
\hline \multicolumn{9}{|c|}{$\begin{array}{l}\text { Late window }(400-1500 \mathrm{~ms}) \\
\text { (c) Prime structure (Active }>\text { Passive) }\end{array}$} \\
\hline Intercept & .94 & .70 & .49 & .48 & 2.78 & 1.39 & 1.89 & .17 \\
\hline Linear & 31.88 & 9.71 & 94.20 & $<.001$ & 32.67 & 9.59 & 91.59 & $<.001$ \\
\hline Quadratic & -33.64 & -10.24 & 104.68 & $<.001$ & -29.65 & -8.69 & 75.37 & $<.001$ \\
\hline Cubic & -19.69 & -5.99 & 35.91 & $<.001$ & -18.43 & -5.40 & 29.15 & $<.001$ \\
\hline \multicolumn{9}{|c|}{ (d) Event similarity (Similar $>$ Dissimilar) $\times$ Prime structure } \\
\hline Intercept & -.63 & -.17 & .03 & .87 & -2.41 & -.67 & .44 & .51 \\
\hline Linear & -34.02 & -5.66 & 31.98 & $<.001$ & -8.20 & -1.29 & 1.65 & .20 \\
\hline Quadratic & -27.07 & -4.50 & 20.22 & $<.001$ & 28.26 & 4.43 & 19.62 & $<.001$ \\
\hline Cubic & 2.82 & .47 & .22 & .64 & -10.43 & -1.63 & 2.67 & .10 \\
\hline
\end{tabular}

(a) Prime Structure

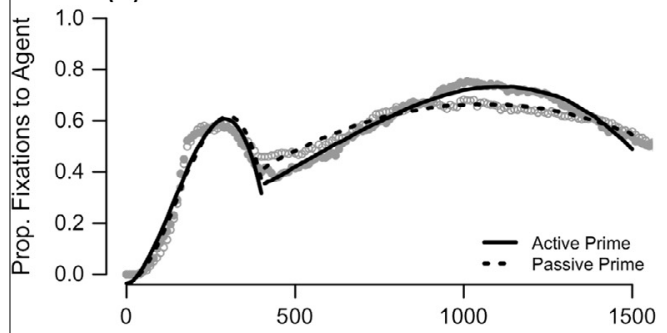

(c) Dissimilar Events

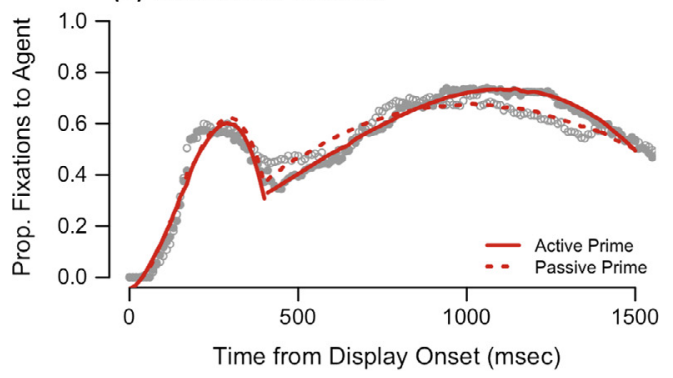

(b) Prime Structure x Event Similarity

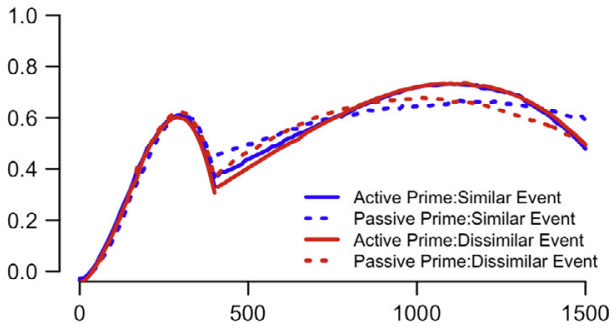

(d) Similar Events

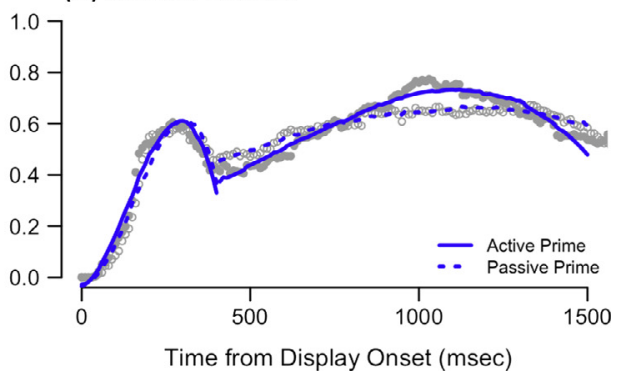

Fig. 7. Data (gray lines) and model fits for active sentences in Experiment 1 (SVO): (a) effects of Prime Structure, (b) interactions between Prime Structure and Event Similarity, and effects of Prime Structure in (c) conceptually dissimilar and (d) similar target events.

reduced when describing similar events (solid and dashed blue lines; linear $\beta=16.11, t=4.18$ ). Second, the quadratic interaction shows that, for conceptually dissimilar events, agent fixations were more peaked following active than passive primes $(\beta=-19.01, t=-4.83$; Fig. $7 \mathrm{c})$. The quadratic structural priming effect for conceptually similar events ( $\beta=-44.33, t=-11.49$ ) was driven by the relatively flat, stable pattern of fixations to the agent after passive primes (Fig. 7d). Thus, structural priming resulted in an increase in late fixations to the agent following active primes, particularly in conceptually dissimilar prime-target event pairs. Conversely, gaze shifts away from the agent after conceptually similar event primes were less affected by the ease of generating a structure than after dissimilar event primes. 


\section{Discussion}

Experiment 1 showed the expected structural priming effect and a strong semantic boost in structural priming: speakers repeated the primed structures more often across conceptually similar than dissimilar prime-target event pairs, and enhancement of priming increased with increased similarity in the prime and target events. Sentence form also depended on Event Codability: the structural primes were more effective in influencing sentence form when the target event was easier to encode as a whole (high event codability). Replicating earlier findings, first fixations had a weak influence on selection of starting points in high codability events.

Timecourse analyses for active sentences showed that formulation was sensitive to the ease of generating active structures in both early and late time windows (Linguistic control of formulation). During early formulation (0$400 \mathrm{~ms}$ ), speakers were more likely to fixate first the agent and then the patient after receiving active primes, consistent with the expectation that selection of a primed active structure should support early encoding of information about two characters instead of one character (Konopka \& Meyer, 2014). During later formulation (post-400 ms), facilitating the generation of an active structure with active primes resulted in slower but more methodical, continued deployment of attention to the subject character, suggesting guidance of linguistic formulation by a linguistic framework.

In contrast, the results show weak evidence of conceptual guidance, as Event Similarity had weaker consequences for formulation than structural priming. The hypothesis that familiarity with the message might facilitate relational encoding during early formulation was not supported (Early Interactivity): exposure to similar prime events did not systematically change the early uptake of information from a target event $(0-400 \mathrm{~ms})$, so the structural primes significantly impacted early formulation in both conceptually similar and dissimilar events. Differential effects of Event Similarity were limited to the later time window (400-1500 ms), with somewhat stronger influences of the structural primes in dissimilar prime-target event pairs (providing weak support for Late Interactivity).

Given the fragility of the influence of event primes on formulation, we examined effects of message familiarity again in Experiment 2 in sentences with a different structure. A possible explanation for the weakness of event primes on formulation in Experiment 1 is that effects of event similarity may be hard to observe in SVO sentences in the current paradigm. Similarity in prime-target event pairs was defined and manipulated by means of similarity of the event action, i.e., information that is expressed with the sentence verb in sentence-medial position. Since formulation is sensitive to linear word order and, as shown here, to manipulations that facilitate production of words in a particular linear order (such as structural priming), early mention of the subject character in SVO sentences may have reduced the possibility of observing gaze differences between event-primed and unprimed sentences.
Experiment 2 tested this hypothesis by examining the formulation of the same event descriptions with VSO/aux-OVS syntax.

\section{Experiment 2}

Dutch sentences beginning with an adjunct (e.g., Today...) require mention of the verb before the event characters. VSO/aux-OVS syntax was thus elicited by presenting prompts like Today and Yesterday before picture onset and asking participants to complete sentences beginning with these prompts.

We first examined whether structural priming in verb-initial sentences differs from priming in verb-medial sentences, and then compared the formulation of active VSO sentences across conditions. If early verb placement results in earlier planning of relational information, then the event primes should have a stronger effect on formulation in Experiment 2 than in Experiment 1 (Joint control of formulation). If, however, formulation is not sensitive to event similarity, Experiment 2 should replicate only the effect of structural primes on eye movements (Linguistic control of formulation).

\section{Method}

\section{Participants}

64 native speakers of Dutch from the same subject pool participated for payment. Four were replaced due to low numbers of scorable target sentences and technical problems.

\section{Materials, design, procedure}

Experiment 2 used the same materials, design, and procedure as Experiment 1 with one exception. At the beginning of each trial, speakers first saw one of four prompt words: Vandaag. .. [Today...], Gisteren... [Yesterday...], op dit moment... [Right now...], In deze afbeelding... [In this picture...]. They then saw a fixation point and, after pressing the space bar, they received a picture in the center of the screen. Their task was to complete the sentence with a description of the pictured event (participants did not repeat the prompt word). On target trials, the prompt word was always Today or Yesterday (counterbalanced across lists).

\section{Scoring and analyses}

The scoring criteria and analyses were the same as in Experiment 1. The final dataset consisted of 1721 sentences (50\% actives, $40 \%$ full passives, $10 \%$ truncated passives).

Event, Agent, and Patient Codability were estimated as in Experiment 1. The three codability indices were not inter-correlated (all $r s<.28, n s$ ) and did not differ across conditions. 


\section{Results}

\section{Structure choice in verb-initial sentences}

Speakers produced a smaller overall proportion of active sentences in Experiment 2 than Experiment 1 (.56 vs. .69; Fig. $3 c$ vs. Fig. 3a), suggesting that active verb-initial sentences were more difficult to generate than passive verb-initial sentences. This is likely because active syntax (VSO) requires that speakers produce the sentence verb first, while passive syntax (aux-OVS) allows speakers to begin sentences with an auxiliary and postpone encoding of the main verb (the verb is produced after the first character as in verb-medial OVS sentences). A stronger preference for passive syntax in Experiment 2 than Experiment 1 thus shows that speakers may have strategically tried to reduce processing load by selecting a structure that allowed minimal early verb planning at the outset of formulation.

\section{Semantic boost in structural priming}

The form of target sentences (actives vs. full passives) was again influenced by the structural primes (Fig. 3c): speakers were more likely to produce active sentences after active than passive primes $(\beta=.54, z=3.32)$. There was a $6 \%$ and $7 \%$ priming effect in conceptually similar and dissimilar prime-target event pairs respectively, so Event Similarity did not interact with Prime Structure $(\beta=.13, z=.38)$. Analyses carried out using the Prime-target Similarity ratings from the no-context and the one-sentence context norming studies (see Fig. 3d for the latter) showed weak effects of prime-target similarity on the magnitude of structural priming $(z=2.50$ and $z=1.53, p=.13$, for the interaction of Prime Structure with the two types of ratings).

\section{Production of full passives and truncated passives}

As in Experiment 1, speakers produced more full passives (and conversely, fewer truncated passives) after passive primes than active primes. Full passives were again produced at comparable rates after conceptually dissimilar passive and active primes (.74 vs. .73), but were produced more often after similar passive primes than similar active primes (.79 vs. .72). This resulted in an interaction of Event Similarity with Prime Structure, $\beta=1.73, z=3.05(z=1.20$, $n s$, and $z=1.89, p=.059$, for the same interaction in models run with the two types of Prime-target Similarity ratings).

\section{Priming effects across different event types}

Speakers again repeated the structure of the primes more often when describing higher-codability than lower-codability target events (priming effects of $13 \%$ and $0 \%$ respectively; Fig. 4b). The interaction of Event Codability with Prime Structure was marginally reliable $(z=1.75, p=.08 ; z=1.80, p=.07 ; z=2.17$, in analyses with Event Similarity and the two types of Prime-Target Similarity ratings).

A weak semantic boost was observed only in higher-codability events. There was a $9 \%$ and $12 \%$ priming effect after conceptually dissimilar and similar primes respectively in higher-codability events; structural priming (and thus also the semantic boost) was weaker in lower-codability events ( $1 \%$ priming after dissimilar and similar primes). However, the three-way interaction of Prime Structure, Event Similarity and Event Codability did not reach significance.

Effects of Agent and Patient Codability on sentence form were considered separately. Speakers produced more actives to describe events with easier-to-name than harder-to-name agents (.61 vs. .54) and more passives to describe events with easier-to-name than harder-to-name patients (.45 vs. .41). The structural primes produced a priming effect only in items with hard-to-name agents, resulting in a weak interaction of Prime Structure with Agent Codability $(z=1.94, p=.053)$. Effects of Patient Codability did not reach significance.

\section{Speech onsets}

Speech onsets were shorter in passive than active sentences (1864 vs.1930 ms; $\beta=93, t=2.64$, for the main effect of Sentence Type). This is consistent with the observation that active sentences beginning with a full verb (VSO) were harder to formulate than passive sentences beginning with an auxiliary (aux-OVS).

Speech onsets were compared for each sentence type individually with respect to Prime Structure, Event Similarity, Event Codability, and Agent or Patient Codability. There were no effects of Prime Structure or Event Similarity. In active sentences, there was an interaction of Event Codability with Agent Codability $(\beta=125$, $t=2.11$ ): speech onsets were shortest in sentences describing high-codability events with high-codability agents, and longer in all remaining cases. In passive sentences, speech onsets varied only with Patient Codability $(\beta=114$, $t=2.19$ ), with faster onsets for sentences with easy-to-name than harder-to-name patients.

\section{Timecourse of formulation}

\section{First fixations and sentence form}

Speakers directed more first fixations to agents than patients (.59 vs. .41), and again, the distribution of first fixations did not differ across conditions. Unlike Experiment 1 , first fixations did not predict structure choice (.56 vs. .55 actives after agent and patient first fixations). There were no interactions with Prime Structure, Event Similarity or Event Codability.

\section{Timecourse}

Timecourse analyses on agent-directed fixations were carried out in two time windows: early (0-400 ms) and late (400-2000 ms). Since speakers maintained fixations on the agent until approximately $2000 \mathrm{~ms}$ (i.e., speech onset), a wider time window was selected for the analysis of late fixations than in Experiment 1.

Both types of primes affected the formulation of VSO sentences (Fig. $6 \mathrm{c}$ and d). The structural primes again influenced the distribution of fixations in the early time 
windows (Table 2a): there was a greater rise (positive linear slope) in agent fixations that was less transient (negative cubic) following active primes than passive primes.

Importantly, in contrast to Experiment 1, effects of Event Similarity were observed from the outset of formulation. In the early window (0-400 ms), interactions of Prime Structure with Event Similarity showed that effects of structural primes were observed primarily in conceptually dissimilar events and were much smaller in similar events (see the linear and quadratic effects in Table $2 \mathrm{~b}$ ). The GCA model fits of the agent-fixation data are shown in Fig. 8a. To clarify the directionality of the interactions, sub-model tests of the by-subject models again examined the effect of the structural primes separately for the conceptually dissimilar and similar event prime conditions (Fig. 8b; only the cubic term interaction was reliable across items). All effects listed below were reliable at $p<.0001$, unless noted otherwise.

When describing conceptually dissimilar target events (Fig. 8c), in the early time window, there was a greater rise in fixations to the agent (greater slope, linear $\beta=28.04$, $t=8.62$ ) that remained elevated (less peaked, quadratic $\beta=10.77, t=3.31$ ) following active compared to passive primes. However, for conceptually similar events (Fig. 8d), these effects were weaker and reversed for the quadratic (linear $\beta=4.30, t=1.36, n s$; quadratic $\beta=-7.01$, $t=-2.22$ ). Thus, repetition of an active structure from primes to targets resulted in more sustained fixations to the agent only in dissimilar events.

Comparable effects of the structural primes and event primes were observed in the later window (400$2000 \mathrm{~ms}$ ). Fixations to the agent increased (positive linear effect) more quickly following active than passive primes (Table 2c; Fig. 8a). Thus, the availability of a linguistic framework after hearing active primes supported continued deployment of attention to the subject character. In contrast to the first time window, however, the pattern of agent-fixations was flatter following active primes (positive quadratic effect). This is consistent with word order: speakers tend to distribute their attention between agents and patients when encoding relational, verb-specific information (Konopka, 2013), so a reduction in agent-directed fixations suggests that speakers may have devoted relatively more resources to encoding the verb than the agent after active primes.

Again, interactions of Prime Structure with Event Similarity showed a smaller effect of structural primes in conceptually similar than dissimilar events (negative quadratic; Table 2d). To clarify the directionality of the interactions, sub-model tests of the by-subject models examined the effect of the structural primes separately for the dissimilar and similar events (Fig. 8b; note that only the quadratic term interaction was reliable across items).

When describing conceptually dissimilar events (Fig. 8c), fixations to the agent were less peaked (quadratic $\beta=95.23, t=20.98$ ) following active compared to passive primes. More specifically, there were generally fewer fixations to the agent after active primes than passive primes in the middle of this time window (800-1500 ms), but speakers then continued fixating the agent longer after active primes (1500-2000 ms). This suggests, again, that easier generation of an active structure resulted in speakers devoting more resources to encoding of the verb and then the sentence subject (the agent). Importantly, this effect was substantially weaker in conceptually similar events (quadratic $\beta=16.80, t=3.98$; Fig. $8 \mathrm{~d}$ ).

\section{Discussion}

Experiment 2 replicated the main findings of Experiment 1 in sentences with a new, non-canonical word order (VSO/aux-OVS). Overall, verb-initial sentences were more difficult to formulate than verb-medial sentences, as shown by speakers' frequent selection of computationally simpler passive syntax. This is a notable contrast with the strong preference to use active syntax over passive syntax in verb-medial sentences and is consistent with the relative scarcity of verb-initial languages (Dryer \& Haspelmath, 2013).

As in Experiment 1, speakers also repeated the structure of the primes when generating target descriptions. This is the first demonstration of structural priming in verb-initial sentences, showing that abstract structures generalize across sentences regardless of verb position. However, the semantic boost was much weaker than in Experiment 1 (a strong effect of Event Similarity on structure choice was observed only in passive sentences, with speakers producing more full passives than truncated passives to describe similar events). Structural priming (with a weak semantic boost) was observed only in higher-codability events, confirming that event codability may be a critical mediating factor in the message-to-language mapping: syntactic structures generalize more easily across sentences when speakers are able to quickly encode a conceptual framework for the target event.

More importantly, verb placement, event similarity and structural priming jointly influenced the timecourse of formulation. First, compared to Experiment 1, early verb placement resulted in different scan paths in active sentences. The first $400 \mathrm{~ms}$ of picture viewing were relatively similar in SVO and VSO sentences, but after $400 \mathrm{~ms}$, speakers maintained fixations on the agent until speech onset in VSO sentences, whereas they shifted their gaze away from the agent much earlier in SVO sentences. This difference in the timing of encoding of the agent is consistent with the word order differences of SVO and VSO sentences.

Second, timecourse analyses revealed that formulation was sensitive to the ease of generating active structures in both early and late time windows, and, unlike Experiment 1, effects of the structural primes were modulated by Event Similarity (Joint control of formulation). In the $0-400 \mathrm{~ms}$ time window, speakers demonstrated a more sustained pattern of agent fixations following active primes in conceptually dissimilar prime-target event pairs. After $400 \mathrm{~ms}$, there was a smaller, though sustained, peak in fixations to the agent after active primes: this suggests that the early availability of an active structure first supported encoding of the verb and then encoding of the agent, demonstrating guidance of linguistic formulation by a linguistic framework. This pattern was observed primarily in conceptually dissimilar events and was much 
Table 2

Experiment 2: GCA results for active sentences in early and late time windows (0-400 ms, 400-2000 ms). (a-c) Polynomial $\times$ Prime Structure: estimates compare the pattern of agent-directed fixations following active vs. passive primes. (b-d) Polynomial $\times$ Prime Structure $\times$ Event Similarity: estimates show how effects of structural primes in (a) differ between similar and dissimilar event conditions.

\begin{tabular}{|c|c|c|c|c|c|c|c|c|}
\hline \multirow[b]{2}{*}{ Effect } & \multicolumn{4}{|c|}{ By-participants } & \multicolumn{4}{|l|}{ By-items } \\
\hline & $\bar{\beta}$ & $t$ & $\chi^{2}$ & $p$ & $\bar{\beta}$ & $t$ & $\chi^{2}$ & $p$ \\
\hline \multicolumn{9}{|c|}{$\begin{array}{l}\text { Early window }(0-400 \mathrm{~ms}) \\
\text { (a) Prime structure (Active }>\text { Passive) }\end{array}$} \\
\hline Intercept & 2.00 & 1.13 & 1.26 & .26 & -1.28 & -.75 & .56 & .45 \\
\hline Linear & 16.23 & 5.33 & 28.32 & $<.001$ & 2.87 & 1.04 & 1.08 & .30 \\
\hline Quadratic & 1.88 & .62 & .38 & .54 & 4.59 & 1.67 & 2.77 & .10 \\
\hline Cubic & -9.77 & -3.21 & 10.29 & $<.001$ & -4.37 & -1.58 & 2.51 & .11 \\
\hline \multicolumn{9}{|c|}{ (b) Event similarity (Similar $>$ Dissimilar) $\times$ Prime structure } \\
\hline Intercept & .38 & .08 & .007 & .93 & -1.39 & -.37 & .14 & .71 \\
\hline Linear & -22.64 & -4.39 & 19.22 & $<.001$ & -20.65 & -4.13 & 17.02 & $<.001$ \\
\hline Quadratic & -20.55 & -3.98 & 15.84 & $<.001$ & -10.87 & -2.18 & 4.73 & .03 \\
\hline Cubic & 5.56 & 1.08 & 1.16 & .28 & 25.57 & 5.12 & 26.12 & $<.001$ \\
\hline \multicolumn{9}{|c|}{$\begin{array}{l}\text { Late window }(400-2000 \mathrm{~ms}) \\
\text { (c) Prime structure (Active }>\text { Passive) }\end{array}$} \\
\hline Intercept & -.93 & -.50 & .25 & .62 & -3.01 & -1.51 & 2.21 & .13 \\
\hline Linear & 22.06 & 6.11 & 37.32 & $<.001$ & -.51 & -.15 & .02 & .88 \\
\hline Quadratic & 53.65 & 14.86 & 220.28 & $<.001$ & 3.18 & .92 & .85 & .36 \\
\hline Cubic & -1.66 & -.46 & .21 & .65 & -4.47 & -1.29 & 1.68 & .20 \\
\hline \multicolumn{9}{|c|}{ (d) Event similarity (Similar $>$ Dissimilar) $\times$ Prime structure } \\
\hline Intercept & -2.97 & -.86 & .73 & .39 & -.51 & -.17 & .03 & .86 \\
\hline Linear & -1.02 & -.15 & .02 & .88 & 8.11 & 1.24 & 1.55 & .21 \\
\hline Quadratic & -79.51 & -11.17 & 138.25 & $<.001$ & -23.12 & -3.55 & 12.59 & $<.001$ \\
\hline Cubic & -3.11 & -.46 & .21 & .64 & 13.50 & 2.07 & 4.30 & .04 \\
\hline
\end{tabular}

(a) Prime Structure

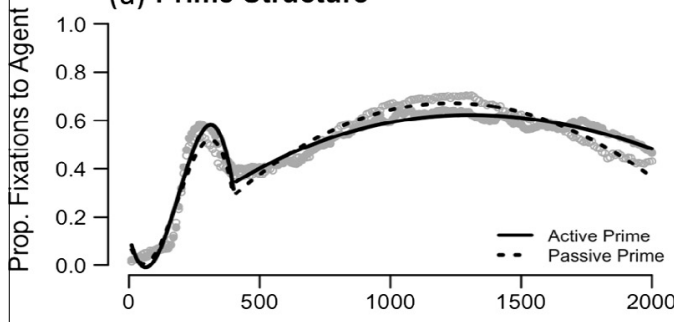

(c) Dissimilar Events

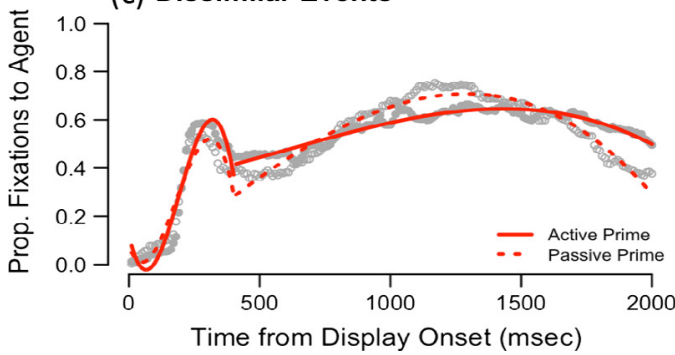

(b) Prime Structure $x$ Event Similarity

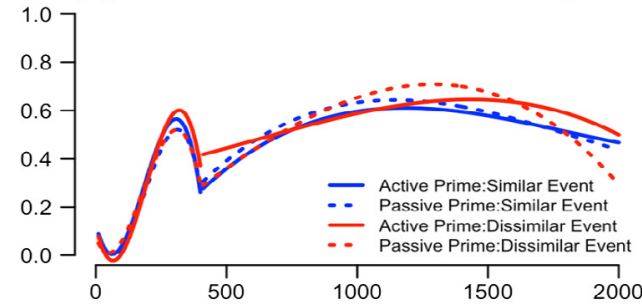

(d) Similar Events

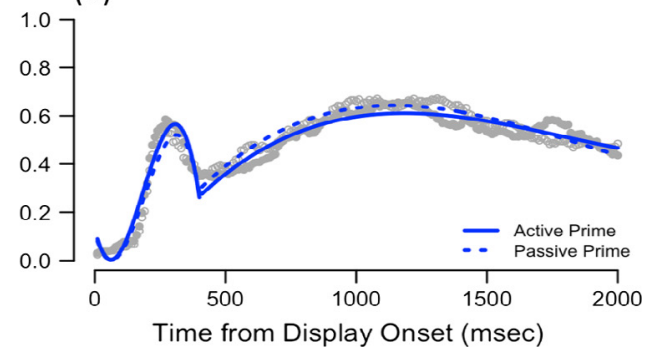

Fig. 8. Data (gray lines) and model fits for active sentences in Experiment 2 (VSO): (a) effects of Prime Structure, (b) interactions between Prime Structure and Event Similarity, and effects of Prime Structure in (c) conceptually dissimilar and (d) similar target events.

weaker in similar events, suggesting that formulating descriptions of familiar events is less likely to depend on the availability of a linguistic framework. Overall, the results show that message similarity can facilitate relational encoding during both early and late formulation (Early and Late Interactivity), and, importantly, that message-level similarity constrains the extent to which linguistic guidance is relevant for formulation.

\section{General discussion}

This paper describes the first experimental investigation into how similarity in the content of two messages (events) influences the formulation of their linguistic descriptions. Experience-dependent changes in the timecourse of formulation were used to assess dependencies between message-level and sentence-level processes. 
Both experiments first verified whether processing of conceptually similar prime events influenced the form of target sentences, and then tested how manipulations of message-level and sentence-level encoding influenced online formulation.

\section{Similarity effects on sentence form}

In Experiment 1, event similarity had a strong impact on the form of target descriptions, demonstrating the equivalent of a semantic boost shown earlier with repetition of semantically related nouns (Cleland \& Pickering, 2003). Since the activation of related concepts is assumed to be responsible for the enhancement of priming, the results show that speakers easily recognized the similarity of prime-target event actions. Experiment 2 showed, for the first time, structural priming in verb-initial sentences but only a weak semantic boost. Current theories of structural processing make no predictions regarding the effects of verb placement on structural generalization. However, verb placement is a critical parameter of phrasal structures in many syntactic theories, and as shown here, it can have implications for the magnitude of the semantic boost. Reasons for this finding are an interesting question for future research. One possibility is that because early verb production inherently requires a high degree of relational encoding at the outset of formulation, activation of conceptually similar action information does not further bias structure selection.

Of course, the semantic boost is only a coarse measure of conceptual overlap in prime-target event pairs. So, to what extent speakers' perceptions of event similarity in both experiments were based on similarities in the relational content of prime-target event pairs? It is possible, for example, that the semantic boost occurred merely because of co-activation of related verb nodes at the conceptual level (a "verb-priming" account), rather than because prime and target events were encoded as conceptually similar events (an "event-priming" account).

We believe that verb-priming alone is an unlikely explanation for the semantic boost. To begin with, a strictly "verb-priming" account may be difficult to distinguish from an "event-priming" account because of the nature of verb representations. Verbs express relational information: by definition, encoding of a verb implies encoding of an event structure that specifies the roles played by characters in an event and the relationship between them (e.g., Fisher, Gleitman, \& Gleitman, 1991). This information is necessary for encoding an event category (e.g., a tripping vs. a pushing event) and may, on different accounts, constitute encoding of event gist (also see Hafri et al., 2013).

Evidence against a "verb-priming" explanation of the semantic boost also comes from two other findings. First, the similarity in the relationship between characters in prime and target events was expressed with a conceptually similar verb, but did not hinge on selection of a specific verb. Results from the norming studies showed that speakers recognized the similarity of prime-target event pairs even in the absence of linguistic cues: there were large differences in ratings for conceptually similar and dissimilar events in the one-sentence context study (where prime events were accompanied by a sentence), but participants also judged similar events as being more related to one another than dissimilar events in the no-context study. It is of course possible that participants in the no-context study described events covertly and that activation of related verbs influenced their ratings. However, it is unlikely that they applied this strategy consistently, as any potential linguistic influences on similarity ratings appeared to be weaker in the no-context study than in the one-context study. This suggests that we can attribute at least part of the variance in the similarity ratings to participants' identification of similar relational content in prime-target event pairs rather than to priming of individual verbs. In addition, ratings from the no-context study were as strong a predictor of the magnitude of the semantic boost as ratings obtained from the one-sentence context study, supporting the hypothesis that the type of similarity that mattered for production was similarity in event representations.

Second, repetition of sentence form from primes to targets was sensitive to event similarity but also to message-level properties like event codability: structural priming was stronger in higher-codability than lower-codability events (events where the conceptual structure of the event was easier than harder to encode). This result points to a form of compatibility between conceptual and linguistic representations that is relevant for the message-to-language mapping: structural flexibility can be achieved more easily when message-level information is readily accessible. In other words, when fast encoding of relational information in a target event facilitates generation of a conceptual framework, speakers can, in turn, map conceptual information more easily onto a linguistic framework. This mapping likely occurs at a level of encoding where structural procedures are sensitive to conceptual properties of a message (see Bock, Loebell, \& Morey, 1992) rather than at a later point in time when speakers retrieve individual words. This makes it unlikely that repetition of sentence structure in similar events was driven primarily by selection of a conceptually similar verb.

Further, a number of studies have shown that, although structural priming can involve generalization of abstract structures (see Pickering \& Ferreira, 2008, for a review), the process of structural assembly is also sensitive to conceptual similarity in prime and target sentences. Abstract structures have parallels in conceptual representations, so structural priming may involve activation and priming of those representations. In support of this claim, Bunger et al. (2013) provide evidence of event structure priming in descriptions of motion events: speakers in their experiments were more likely to mention path information when describing a target motion event when prime sentences also included path information. This effect was independent of lexical overlap in primes and targets. Although their dependent measure was overt mention of path information, the results show that priming of a linguistic structure may extend to priming of event components (i.e., individual elements of a message). Our analyses comparing production of full passives and truncated passives show a similar effect: target events were described with full 
passives more often after conceptually similar than dissimilar passive primes, suggesting that the event primes influenced encoding of the message-level representations of target events.

\section{Similarity effects in formulation: Linguistic control vs. Joint} control of formulation

Given joint effects of event primes and structural primes on sentence form, we then asked whether and how the primes influenced formulation. As outlined in the Introduction, an account positing purely Linguistic control of formulation predicts that linguistic (structural) similarity from prime to target sentences, but not message similarity, should influence formulation. In contrast, a Joint control of formulation account predicts effects of both linguistic and message similarity. To address this question, we examined whether the timecourse of formulation varied with similarity in the content of prime-target event pairs (message similarity) and with speakers' choice to repeat the structure of prime sentences on target trials (structural similarity) from the first fixation until speech onset. The results showed that message-level effects can impact formulation from its outset, particularly if relational information (i.e., the verb) is to be prepared early on. We discuss the implications of variation in processing at the message level on processing at the sentence level in terms of identifying constraints on incrementality during sentence formulation.

\section{First fixations}

In Experiment 1, the selection of starting points for target descriptions (i.e., the choice between starting a sentence with the agent or the patient) was sensitive to two variables modulating the ease of encoding message-level information: experimentally manipulated event similarity and item-specific event codability. Replicating Kuchinsky and Bock (2010), speakers began their sentences with the first-fixated character more often in lower-codability than higher-codability events: fast encoding of relational information in higher-codability events reduced the likelihood of speakers mentioning event characters in the order of fixation, and, instead, favored selection of starting points on conceptual grounds. Event similarity had an analogous effect, showing that facilitating conceptual encoding can influence structure choice through a similar mechanism. In sum, first fixations in lower-codability events and conceptually less familiar events facilitated selection of a character to be mentioned first (i.e., a word order choice), while in higher-codability events and conceptually more familiar events, speakers were more likely to begin sentences with a character selected to be the sentence subject (i.e., a grammatical category). This finding additionally supports the conclusion that the manipulation of event similarity influenced encoding of an event representation rather than solely boosting activation of semantically related verb nodes.

Interestingly, there was no influence of first fixations, event similarity or event codability on sentence form in Experiment 2. On the assumption that first fixations can facilitate selection of a starting point (the first content word of a sentence), there should indeed be no influence of first fixations on structure choice in sentences where the first content word is not a character name. In addition, encoding of the first content word in verb-initial sentences specifically requires relational encoding (i.e., information that is not specific to one single character), further reducing any influence of early, bottom-up character accessibility on structure choice.

\section{Timecourse of formulation}

Comparing the entire timecourse of formulation across conditions allowed us to gauge generally when, and thus at what level of encoding, message-level and sentence-level variables influence formulation.

Effects of structural primes were observed throughout the formulation of active sentences in both experiments. Speakers were less likely to fixate the agent immediately after picture onset (0-400 ms) in SVO sentences after active primes (Experiment 1 ). Effects of structural primes in VSO sentences were inconsistent across participants and items, and thus remain to be replicated (Experiment 2 ). In the later window in both experiments (post- $400 \mathrm{~ms}$ ), agent fixations rose more slowly and peaked later in structurally primed than unprimed sentences. Together, these differences across conditions suggest that easy generation of an active structure after active primes - and thus early availability of a linguistic framework - increased the likelihood of speakers devoting resources to encoding relational information (information about both characters) before encoding the subject character (Konopka \& Meyer, 2014).

Effects of event primes were observed only during the late window in Experiment 1 (Joint control of formulation: Late Interactivity) but, as expected, were present throughout formulation in Experiment 2 (Joint control of formulation: Early and Late Interactivity). In SVO sentences (Experiment 1), the sustained pattern of fixations to the agent after active primes was stronger in dissimilar than similar target events during later formulation; in VSO sentences (Experiment 2), the more sustained pattern of agent fixations after active primes was observed in conceptually dissimilar target events and only weakly or not at all in similar target events. Thus in both experiments, familiarity with the content of the target events reduced the influence of structural primes on formulation. This shows control of deployment of attention by a message-level framework, and importantly, the direction of these effects suggests that conceptual guidance is a stronger predictor of formulation than linguistic guidance. Put differently, linguistic variables exert an influence on formulation in the absence of top-down conceptual information, but formulation appears to be relatively immune to the ease of linguistic encoding when speakers are more familiar with the target message.

More generally, these results add to the growing body of evidence identifying a hierarchy of variables that shape production. Higher-level variables take precedence over lower-level variables. For example, comparing effects of perceptual, lexical and conceptual variables on sentence form shows that variability at the perceptual level is the weakest predictor, both independently and in conjunction 
with other variables. Effects of perceptual accessibility (inherent accessibility or accessibility manipulated with attention-grabbing cues) are usually operationalized in terms of the relationship between first fixations and sentence form, and are much weaker than effects of sentence-level variables: they are secondary to lexical accessibility and to the availability of sentence structures in determining sentence form (Konopka \& Meyer, 2014). They are also secondary to effects of message-level variables like event codability (Kuchinsky \& Bock, 2010) and, as shown here, familiarity with message content. Likewise, in terms of formulation, sentence-level variables are secondary to message-level variables: lexical accessibility is secondary to event codability (Konopka \& Meyer, 2014; Kuchinsky \& Bock, 2010), and, as shown here, structural availability may be secondary to message familiarity.

This influence of message-level variables on formulation is also consistent with findings from both comprehension and production studies showing strong effects of context on language processing. For example, sentence comprehension in a discourse context benefits from activation (or prediction) of context-relevant information: context reduces referential ambiguity and lexical ambiguity (e.g., Brown-Schmidt, 2009; Brown-Schmidt \& Konopka, 2011; Federmeier, McLennan, De Ochoa, \& Kutas, 2002). Similarly, in production, sentence context facilitates retrieval of expected but infrequent words (Griffin \& Bock, 1998) and it reduces effects of conceptual and lexical accessibility on structure choice (Konopka, 2014). In short, such results suggest that "classic" psycholinguistic effects (e.g., frequency effects) observed at the word level in isolated sentences need not transfer to sentences produced in a larger context. In the current experiments, the prime events can be seen as providing conceptually relevant information (albeit in a very impoverished context) for processing target events, and, similarly to the comprehension and production studies mentioned above, they show that the presence of supporting message-level information can reduce effects of sentence-level variables on formulation. Notably, although both experiments used a controlled experimental paradigm, the types of fluctuations in the ease of message-level encoding that were induced with the event primes are normal in everyday language production. This research thus highlights the importance of going beyond analyses of single sentences and considering message-level influences on language processing to develop theories that are more representative of language use outside of the lab.

\section{Implications of verb placement for formulation}

At the same time, we also note that there may be limits on message-driven changes in formulation because sentence formulation is also strongly controlled by linear word order. The contrast between Experiments 1 and 2 shows that effects of message similarity on formulation are not ubiquitous: the formulation of verb-medial sentences in Experiment 1 was primarily sensitive to changes in the ease of linguistic encoding (consistent with the Linguistic control of formulation account). This was true even though speakers generated event descriptions spontaneously, which requires high engagement of message-level processes at the beginning of formulation. We propose that the difference between Experiment 1 and 2 shows structure-specific allocation of resources to relational and non-relational encoding at different points during formulation: early production of a subject character requires, relatively speaking, more extensive early encoding of non-relational information than does early production of the verb. This type of resource allocation may support efficient encoding, as production can benefit from incremental planning of message and sentence elements in the order of mention and shortly before articulation. In this sense, a basic property of language - linearity - may constrain effects of message-level variables (like event similarity) on formulation.

Further, we note that the influence of event similarity on sentence form was stronger than its influence on formulation (at least in Experiment 1). In contrast, consistent facilitation of formulation by structural primes suggests that, within one message type, formulation is highly sensitive to the efficiency of sentence-level encoding operations. Thus to a large extent, formulation may be controlled by processes that are ultimately responsible for outputting words in a particular order (i.e., structural processes). Cross-linguistic studies provide converging evidence that effects of linguistic structure on formulation such as word order requirements or morphosyntax - can be observed from the outset of formulation (e.g., Hwang \& Kaiser, 2014; Norcliffe et al., 2015; Sauppe et al., 2013). This gives linguistic structure an important role in mediating the message-to-language mapping.

\section{Conclusions}

Identifying systematic sources of variability during formulation in an incremental production system can inform debates on the relationship between message-level and sentence-level processes. The current experiments offer the first insight into how the formulation of simple messages and sentences can change with speakers' experience with the content of a message. The results show that the ease of conceptual and linguistic encoding can jointly determine selection of sentence form as well as the timecourse of formulation. Since familiarity with a message may have stronger implications for what speakers choose to say in more open-ended production "tasks" (i.e., situations resembling every-day conversational contexts), an important goal for future research is to extend the scope of current theories and paradigms to more naturalistic production settings.

\section{Acknowledgments}

We thank Moniek Schaars, Esther Kroese, and Annelies van Wijngaarden for invaluable help constructing stimuli, collecting and processing data, Tilman Harpe for drawing a selection of the pictures, and Antje Meyer for comments on the manuscript. 


\section{Appendix A}

Targets events (with conceptually similar/dissimilar event primes in the active voice in English and Dutch).

\begin{tabular}{|c|c|}
\hline Targets & Primes \\
\hline $\begin{array}{l}\text { 1. Man shooting } \\
\text { woman }\end{array}$ & $\begin{array}{l}\text { The eskimo is } \\
\text { attacking/watching the bear. } \\
\text { (De eskimo vaalt de beer } \\
\text { aan/bekijkt de beer.) }\end{array}$ \\
\hline $\begin{array}{l}\text { 2. Nurse carrying } \\
\text { baby }\end{array}$ & $\begin{array}{l}\text { The workers are } \\
\text { moving/repairing the piano. } \\
\text { (De werkmannen } \\
\text { verplatsen/repareren de piano.) }\end{array}$ \\
\hline $\begin{array}{l}\text { 3. Pirate burying } \\
\text { treasure }\end{array}$ & $\begin{array}{l}\text { The maid is hiding/eating the } \\
\text { chocolate } \\
\text { (Het dienstmeisje verstopt de } \\
\text { chocolade/eet de chocolade op.) }\end{array}$ \\
\hline $\begin{array}{l}\text { 4. Make-up artist } \\
\text { painting model }\end{array}$ & $\begin{array}{l}\text { The barber is } \\
\text { shaving/threatening the prisoner. } \\
\text { (De kapper scheert/bedreigt de } \\
\text { gevangene.) }\end{array}$ \\
\hline 5. Bee stinging man & $\begin{array}{l}\text { The magician is } \\
\text { pinching/hypnotizing the clown. } \\
\text { (De magiër knijpt/hypnotiseert } \\
\text { de clown.) }\end{array}$ \\
\hline $\begin{array}{l}\text { 6. Cop arresting } \\
\text { boy }\end{array}$ & $\begin{array}{l}\text { The cat is catching/licking the } \\
\text { mouse. } \\
\text { (De kat vangt/likt de muis.) }\end{array}$ \\
\hline $\begin{array}{l}\text { 7. Elephant lifting } \\
\text { sultan }\end{array}$ & $\begin{array}{l}\text { The groom is carrying/kissing the } \\
\text { bride. } \\
\text { (De bruidegom draagt/kust de } \\
\text { bruid.) }\end{array}$ \\
\hline $\begin{array}{l}\text { 8. Cop stopping } \\
\text { truck }\end{array}$ & $\begin{array}{l}\text { The boy is trapping/picking up } \\
\text { the ladybugs. } \\
\text { (De jongen vangt/pakt de } \\
\text { lieveheersbeestjes op.) }\end{array}$ \\
\hline $\begin{array}{l}\text { 9. Boxer punching } \\
\text { cheerleader }\end{array}$ & $\begin{array}{l}\text { The tiger is scratching/scaring } \\
\text { the tourist. } \\
\text { (De tijger krabt/maakt de toerist } \\
\text { bang.) }\end{array}$ \\
\hline $\begin{array}{l}\text { 10. Woman } \\
\text { massaging man }\end{array}$ & $\begin{array}{l}\text { The doctor is washing/feeding } \\
\text { the baby. } \\
\text { (De dokter wast/voedt de baby.) }\end{array}$ \\
\hline $\begin{array}{l}\text { 11. Windmill } \\
\text { hitting farmer }\end{array}$ & $\begin{array}{l}\text { The ballerina is slapping/hugging } \\
\text { the pianist. } \\
\text { (De ballerina slaat/omhelst de } \\
\text { pianist.) }\end{array}$ \\
\hline $\begin{array}{l}\text { 12. Dog chasing } \\
\text { mailman }\end{array}$ & $\begin{array}{l}\text { The paparazzo is } \\
\text { following/photographing the } \\
\text { queen. } \\
\text { (De paparazzo volgt/fotografeert } \\
\text { de koningin.) }\end{array}$ \\
\hline $\begin{array}{l}\text { 13. Mother } \\
\text { dressing boy }\end{array}$ & $\begin{array}{l}\text { The man is brushing/examining } \\
\text { the show-dog. } \\
\text { (De man borstelt/onderzoekt de } \\
\text { showhond.) }\end{array}$ \\
\hline $\begin{array}{l}\text { 14. Truck towing } \\
\text { car }\end{array}$ & $\begin{array}{l}\text { The farmer is pulling/letting out } \\
\text { the donkey. }\end{array}$ \\
\hline
\end{tabular}

Appendix A (continued)

\begin{tabular}{|c|c|}
\hline Targets & Primes \\
\hline & (De boer trekt/laat de ezel vrij.) \\
\hline $\begin{array}{l}\text { 15. Journalist } \\
\text { interviewing } \\
\text { actor }\end{array}$ & $\begin{array}{l}\text { The judge is questioning/accusing } \\
\text { the witness. } \\
\text { (De rechter } \\
\text { ondervraagt/beschuldigt de } \\
\text { getuige.) }\end{array}$ \\
\hline 16. Photographer & The lifeguard is \\
\hline filming man & $\begin{array}{l}\text { watching/reviving the child. } \\
\text { (De strandwacht } \\
\text { bekijkt/reanimeert het kind.) }\end{array}$ \\
\hline $\begin{array}{l}\text { 17. Train crushing } \\
\text { bus }\end{array}$ & $\begin{array}{l}\text { The burglar is smashing/opening } \\
\text { the window. } \\
\text { (De inbreker slaat het raam } \\
\text { kapot/opent het raam.) }\end{array}$ \\
\hline 18. Girl tickling boy & $\begin{array}{l}\text { The grandfather is } \\
\text { entertaining/kissing the toddler. } \\
\text { (De opa vermaakt/kust de } \\
\text { peuter.) }\end{array}$ \\
\hline $\begin{array}{l}\text { 19. Ambulance } \\
\text { hitting woman }\end{array}$ & $\begin{array}{l}\text { The lightning is striking/missing } \\
\text { the church. } \\
\text { (De bliksem raakt/mist de kerk.) }\end{array}$ \\
\hline $\begin{array}{l}\text { 20. Shark } \\
\text { swallowing man }\end{array}$ & $\begin{array}{l}\text { The snake is biting/following the } \\
\text { leopard. }\end{array}$ \\
\hline
\end{tabular}

(De slang bijt/volgt het luipard.)

21. Old waiter The girl is throwing

kicking man out away/opening the present.

(Het meisje gooit de cadeau weg/opent de cadeau.)

22. Sprinkler The punk is spraying/damaging splashing old the fence.

woman (De punker spuit/maakt het hek kapot.)

23. Robot smashing The baby is ripping/biting the computer teddy bear.

(De baby verscheurt/bijt de teddybeer.)

24. Girl pushing The dog is guiding/scaring the boy on sled blind man.

(De hond leidt/maakt de blinde man bang.)

25. Mechanic fixing The nurse is bandaging/moving car

the patient

(De verpleegster verbindt/verplaatst de patient.)

\section{Swimmer pushing paparazzo}

\section{Bulldozer destroying building}
28. Doctor examining patient

The hippie is tripping/paying the waiter.

(De hippie tackelt/betaalt de ober.)

The detective is burning/finding the drugs.

(De detective verbrandt/vindt de drugs.)

The security officer is inspecting/taking away the suitcase.

(De beveiligsman 
Appendix A (continued)

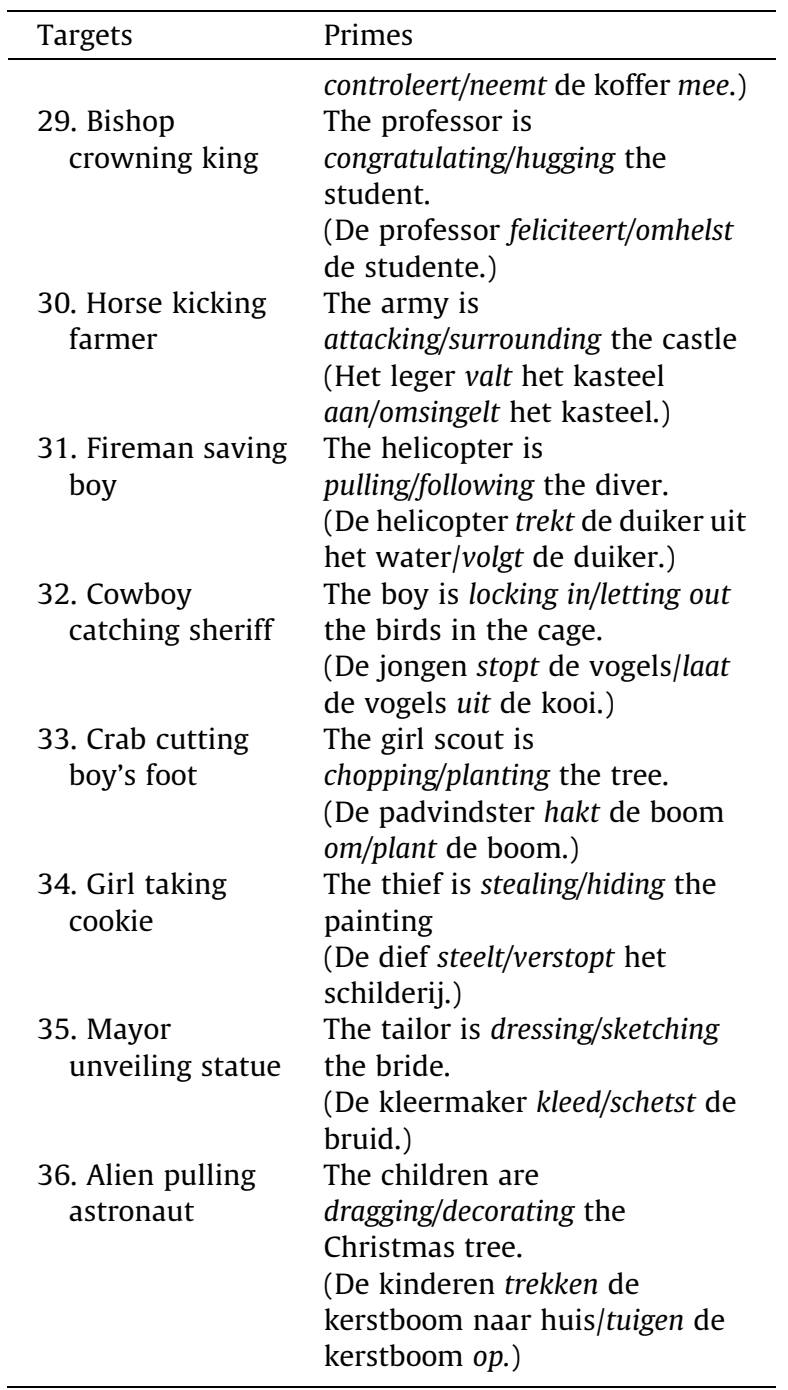

\section{References}

Arnold, J. E. (2010). How speakers refer: The role of accessibility. Language and Linguistics Compass, 4, 187-203.

Baayen, R. H., Davidson, D. J., \& Bates, D. M. (2008). Mixed-effects modeling with crossed random effects for subjects and items. Journal of Memory and Language, 59, 390-412.

Barr, D. J. (2008). Pragmatic expectations and linguistic evidence: Listeners anticipate but do not integrate common ground. Cognition, 109, 18-40.

Barr, D. J., Levy, R., Scheepers, C., \& Tily, H. J. (2013). Random effects structure for confirmatory hypothesis testing: Keep it maximal. Journal of Memory and Language, 68, 255-278.

Bates, D., Maechler, M., Bolker, B., \& Walker, S. (2014). Ime4: Linear mixedeffects models using Eigen and S4. $R$ package version 1.1-7. <http:| CRAN.R-project.org/package=lme4>.

Bock, K. (1982). Toward a cognitive psychology of syntax: Information processing contributions to sentence formulation. Psychological Review, 89, 1-47.

Bock, J. K. (1986). Syntactic persistence in language production. Cognitive Psychology, 18, 355-387.

Bock, J. K., Irwin, D. E., \& Davidson, D. J. J. (2004). Putting first things first. In F. Ferreira \& M. Henderson (Eds.), The integration of language, vision, and action: Eye movements and the visual world (pp. 249-278). New York: Psychology Press.

Bock, K., Loebell, H., \& Morey, R. (1992). From conceptual roles to structural relations: Bridging the syntactic cleft. Psychological Review, 99, 150-171.

Brown-Schmidt, S., \& Konopka, A. E. (2011). Experimental approaches to referential domains and the on-line processing of referring expressions in unscripted conversation. Information, 2, 302-326.

Brown-Schmidt, S. (2009). Partner-specific interpretation of maintained referential precedents during interactive dialog. Journal of Memory and Language, 61, 171-190.

Brown-Schmidt, S., \& Konopka, A. E. (2008). Little houses and casas pequeñas: Message formulation and syntactic form in unscripted speech with speakers of English and Spanish. Cognition, 109, 274-280.

Brown-Schmidt, S., \& Konopka, A. E. (2014). Processes of incremental message planning during conversation. Psychonomic Bulletin $\mathcal{E}$ Review. http://dx.doi.org/10.3758/s13423-014-0714-2.

Bunger, A., Papafragou, A., \& Trueswell, J. (2013). Event structure influences language production: Evidence from structural priming in motion event description. Journal of Memory and Language, 69, 299-323.

Chang, F., Dell, G. S., \& Bock, K. (2006). Becoming syntactic. Psychological Review, 113, 234-272.

Chang, F., \& Fitz, H. (2014). Computational models of sentence production: A Dual-path approach. In V. Ferreira, M. Goldrick, \& M. Miozzo (Eds.), The Oxford handbook of language production (pp. 70-87). Oxford University Press.

Cleland, A. A., \& Pickering, M. J. (2003). The use of lexical and syntactic information in language production: Evidence from the priming of noun-phrase structure. Journal of Memory and Language, 49, 214-230.

Clifton, C., Jr., Meyer, A., Wurm, L., \& Treiman, R. (2012). Language comprehension and production. In A. F. Healy \& R. W. Proctor (Eds.). Comprehensive handbook of psychology (Vol. 4, pp. 523-547). New York: Wiley.

Dell, G. S. (1986). A spreading-activation theory of retrieval in sentence production. Psychological Review, 93, 283-321.

Dell, G. S., \& O’Seaghdha, P. G. (1992). Stages of lexical access in language production. Cognition, 42, 287-314.

Dobel, C., Gumnior, H., Bolte, J., \& Zwitserlood, P. (2007). Describing scenes hardly seen. Acta Psychologica, 125, 129-143.

Dryer, M. D., \& Haspelmath, M. (2013). The world atlas of language structures online. Leipzig: Max Planck Institute for Evolutionary Anthropology. <http://wals/info>.

Federmeier, K. D., McLennan, D. B., De Ochoa, E., \& Kutas, M. (2002). The impact of semantic memory organization and sentence context information on spoken language processing by younger and older adults: An ERP study. Psychophysiology, 39, 133-146.

Ferreira, F., \& Swets, B. (2002). How incremental is language production? Evidence from the production of utterances requiring the computation of arithmetic sums. Journal of Memory and Language, 46, 57-84.

Fisher, C., Gleitman, H., \& Gleitman, L. R. (1991). On the semantic content of subcategorization frames. Cognitive Psychology, 23, 331-392.

Gleitman, L., January, D., Nappa, R., \& Trueswell, J. C. (2007). On the give and take between event apprehension and utterance formulation. Journal of Memory and Language, 57, 544-569.

Grice, H. P. (1975). Logic and Conversation. In P. Cole \& Jerry L. Morgan (Eds.). Speech Acts (vol. 3). New York: Academic Press.

Griffin, Z. M. (2001). Gaze durations during speech reflect word selection and phonological encoding. Cognition, 82, B1-B14.

Griffin, Z. M., \& Bock, K. (1998). Constraint, word frequency, and levels of processing in spoken word production. Journal of Memory and Language, 38, 313-338.

Griffin, Z. M., \& Bock, K. (2000). What the eyes say about speaking. Psychological Science, 11, 274-279.

Griffin, Z. M. (2004). Why look? Reasons for eye movements related to language production. In F. Ferreira \& J. Henderson (Eds.), The integration of language, vision, and action: Eye movements and the visual world (pp. 213-247). New York: Psychology Press.

Hafri, A., Papafragou, A., \& Trueswell, J. C. (2013). Getting the gist of events: Recognition of two-participant actions from brief displays. Journal of Experiment Psychology: General, 142, 880-905.

Hartsuiker, R. J., Bernoley, S., Schoonbaert, S., Speybroeck, S., \& Vanderelst, D. (2008). Syntactic priming persists while the lexical boost decays: Evidence from written and spoken dialogue. Journal of Memory and Language, 58, 214-238.

Hwang, H., \& Kaiser, E. (2014). The role of the verb in grammatical function assignment in English and Korean. Journal of Experimental Psychology: Learning, Memory and Cognition, 40, 1363-1376. 
Jaeger, T. F. (2008). Categorical data analysis: Away from ANOVAs (transformation or not) and towards logit mixed models. Journal of Memory and Language, 59, 434-446.

Jaeger, T. F. (2010). Redundancy and reduction: Speakers manage syntactic information density. Cognitive Psychology, 61, 23-62.

Kalénine, S., Mirman, D., \& Buxbaum, L. J. (2012). A Combination of Thematic and Similarity-Based Semantic Processes Confers Resistance to Deficit Following Left Hemisphere Stroke. Frontiers in Human Neuroscience, 6, 106. http://dx.doi.org/10.3389/fnhum.2012.00106.

Kalénine, S., Mirman, D., Middleton, E. L., \& Buxbaum, L. J. (2012). Temporal dynamics of activation of thematic and functional knowledge during conceptual processing of manipulable artifacts. Journal of Experimental Psychology: Learning, Memory, and Cognition, 38, 1274-1295.

Konopka, A. E. (2012). Planning ahead: How recent experience with structures and words changes the scope of linguistic planning. Journal of Memory and Language, 66, 143-162.

Konopka, A. E. (2013). Discourse changes the timecourse of sentence formulation. Poster presented at the 19th AMLaP conference, Marseille, France.

Konopka, A. E. (2014). Top-down context effects on sentence production. Paper presented at the April meeting of the Experimental Psychology Society [EPS], Kent, UK.

Konopka, A. E., \& Brown-Schmidt, S. (2014). Message encoding. In M. Goldrick, V. Ferreira, \& M. Miozzo (Eds.), The Oxford Handbook of Language Production (pp. 3-20). Oxford University Press.

Konopka, A. E., \& Meyer, A. S. (2014). Priming sentence planning. Cognitive Psychology, 73, 1-40.

Kuchinsky, S. E. (2009). From seeing to saying: Perceiving, planning producing. Unpublished doctoral dissertation, University of Illinois at Urbana-Champaign.

Kuchinsky, S. E., Ahlstrom, J. B., Vaden, K. I., Cute, S. L., Humes, L. E., Dubno, J. R., et al. (2013). Pupil size varies with word listening and response selection difficulty in older adults with hearing loss. Psychophysiology, $50,23-34$.

Kuchinsky, S. E., \& Bock, K. (2010). From seeing to saying: Perceiving, planning, producing. Paper presented at the 23rd CUNY human sentence processing conference, New York.

Lee, E., Brown-Schmidt, S., \& Watson, D. G. (2013). Ways of looking ahead: Hierarchical planning in language production. Journal of Memory and Language, 129, 544-562.

Levelt, W. J. M., Roelofs, A., \& Meyer, A. S. (1999). A theory of lexical access in speech production. Behavioral and Brain Sciences, 22, 1-75.

Levelt, W. J. M. (1989). Speaking: From intention to articulation. Cambridge, MA: MIT Press.

Meyer, A. S., Sleiderink, A. M., \& Levelt, W. J. M. (1998). Viewing and naming objects: Eye movements during noun phrase production. Cognition, 66, B25-B33.

Mirman, D. (2014). Growth curve analysis and visualization using R. Boca Raton, FL: Chapman \& Hall/CRC.

Mirman, D., Dixon, J. A., \& Magnuson, J. S. (2008). Statistical and computational models of the visual world paradigm: Growth curves and individual differences. Journal of Memory and Language, 59, 475-494.

Nakamura, C., Arai, M., \& Mazuka, R. (2012). Immediate use of prosody and context in predicting a syntactic structure. Cognition, 125, 317-323.

Norcliffe, E., Konopka, A. E., Brown, P., \& Levinson, S. (2015). Word order affects the timecourse of sentence formulation in Tzeltal. Language, Cognition and Neuroscience.

Pickering, M. J., \& Branigan, H. (1998). The representation of verbs: Evidence from syntactic priming in language production. Journal of Memory and Language, 39, 633-651.

Pickering, M. J., \& Ferreira, V. S. (2008). Structural priming: A critical review. Psychological Bulletin, 134, 427-459.

Pickering, M. J., \& Garrod, S. (2004). The interactive-alignment model: Developments and refinements. Behavioral and Brain Sciences, 27, 212-225.

$\mathrm{R}$ Core Team (2014). R: A language and environment for statistical computing. R Foundation for Statistical Computing, Vienna, Austria. <http://www.R-project.org/>.

Rapp, B., \& Goldrick, M. (2000). Discreteness and interactivity in spoken word production. Psychological Review, 107, 460-499.

Roelofs, A. (1997). The WEAVER model of word-form encoding in speech production. Cognition, 64, 249-284.

Santestban, M., Pickering, M. J., \& McLean, J. F. (2010). Lexical and phonological effects on syntactic processing: Evidence from syntactic priming. Journal of Memory and Language, 63, 347-366.

Sauppe, S., Norcliffe, E., Konopka, A. E., van Valin, R. D., \& Levinson, S. (2013). Dependencies first: Eye-tracking evidence from sentence production in Tagalog. Paper presented at the 35th meeting of the Cognitive Science Society, Berlin, Germany, July 2013.

Schober, M. F., \& Clark, H. H. (1989). Understanding by addressees and overhearers. Cognitive Psychology, 21, 211-232.

Tooley, K., Konopka, A. E., \& Watson, D. G. (2014). Can prosodic structure be primed (like syntactic structure)? Journal of Experimental Psychology: Learning, Memory, and Cognition, 40, 348-363.

Von Stutterheim, C., Flecken, M., \& Carroll, M. (2013). Introduction: Conceptualizing in a second language. International Review of Applied Linguistics in Language Teaching, 51.

Wagner, V., Jescheniak, J. D., \& Schriefers, H. (2010). On the flexibility of grammatical advance planning during sentence production: Effects of cognitive load on multiple lexical access. Journal of Experimental Psychology: Learning, Memory, and Cognition, 36, 423-440.

Wagner, M., \& Watson, D. G. (2010). Experimental and theoretical advances in prosody: A review. Language and Cognitive Processes, 25, 905-945.

Wheeldon, L. (2013). Producing spoken sentences: The scope of incremental planning. In P. Perrier, \& P. Lang Verlag (Eds.), Cognitive and physical models of speech production, speech perception, and production-perception interaction.

Wilkes-Gibbs, D., \& Clark, H. H. (1992). Coordinating beliefs in conversation. Journal of Memory and Language, 31, 183-194. 